\title{
Magmatism in the Garrett transform fault (East Pacific Rise near $13^{\circ} 27^{\prime}$ 'S)
}

\author{
Roger Hékinian and Daniel Bideau \\ Department of Marine Geosciences, IFREMER (Institut Français de Recherche pour l'Exploitation de la Mer) \\ Plouzané, France
}

Réjean Hébert

Département de Géologie, Université Laval, Québec, Canada

\section{Yaoling Niu}

Department of Earth Sciences, University of Queensland, Brisbane, Australia

\begin{abstract}
The Garrett transform is characterized by recent (zero age) volcanic activity located within the active tectonic domain of the transform valley at depths greater than the $3500 \mathrm{~m}$. This intratransform volcanic activity contributed to the formation of constructional edifices forming ridges ( $>300 \mathrm{~m}$ in height) and small mounds ( $<20 \mathrm{~m}$ in height) built near slivers of serpentinized peridotites. The erupted lavas are depleted mid ocean ridge basalts (MORBs) with low ratios of K/Ti (0.02-0.11), Zr (30-100 ppm), Y (18-50 ppm), and (La/Sm) $N(0.25-0.60)$. Their more depleted nature and smaller range of variability for the compatible elements $(\mathrm{Ni}=70-180 \mathrm{ppm}$,

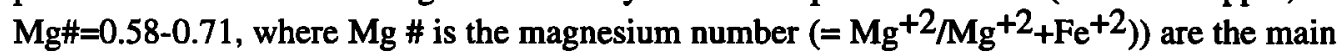
points of difference between the Garrett intratransform volcanics (GITV) and those from the ultrafast south East Pacific Rise (SEPR). However, ferrobasalts (Mg\#=0.41-0.55) were collected from the intratransform walls as well as at the EPR-transform intersection. The GITV are even more depleted in incompatible elements than lava from the north East Pacific Rise $\left(21^{\circ} \mathrm{N}-11^{\circ} 26^{\prime} \mathrm{N}\right)$. The Garrett recent lava is believed to have erupted after the successive, incremental partial melting and discontinuous melt extraction of a composite lherzolitic mantle similar to that of the SEPR. The limited range of incompatible element ratios $\left(\mathrm{Zr} / \mathrm{Y}=1-2.5,(\mathrm{Ce} / \mathrm{Yb})_{N}=0.4-1\right)$ and $\mathrm{K} / \mathrm{Ti}$ ratios $(<0.14)$ and the samples more porphyritic nature with respect to other SEPR rocks suggest that the intratransform volcanics from the Garrett are extracted from their source and channeled directly toward the surface without extensive mixing in magma chambers. In order to explain the restricted range of compositional variabilities and the absence of the enriched basalts produced by prior melting, we postulate that even though they were produced, these most enriched end-member lavas did not reach the surface; instead, we propose these melts contribute to the formation of impregnated mantle material in the lithosphere. We suggest that this same petrogenetic style of intratransform volcanism might also characterize other oceanic provinces associated with low magmatic to quasiamagmatic regimes.
\end{abstract}

\section{Introduction}

The Garrett transform fault is one of only a few fracture zones where recent volcanic activity has been observed. Intratransform volcanism has been previously found the Siqueiros transform (North Pacific) [Natland, 1989; Casey et al., 1991; Perfit et al., 1994], the Saint Paul's Rocks [Melson et al., 1967; 1972; Bonatti, 1990], and some "multiple" fracture zones of the East Pacific rise (e.g., Gofar-Quebrada [Lonsdale, 1978] and Wilkes [Searle, 1983]) but has only been sampled at the Siqueiros and Saint Paul's Rocks.

The Garrett transform fault contains a variety of recent volcanic structures erupted in the transform domain at more than $3500 \mathrm{~m}$ depth (Figures 1a-1c). These structures have formed NE-SW trending oblique ridges as well as east-west

Copyright 1995 by the American Geophysical Union.

Paper number $94 \mathrm{JB} 02125$.

0148-0227/95/94JB-02125\$05.00 trending ridges (300-1000 $\mathrm{m}$ high) with volcanic cones within the transform valley. Smaller mounds ( $<20 \mathrm{~m}$ high) and sporadic, more recent lava flows invading older terrain were also detected in the deepest part of the transform troughs and walls (3500-5000 $\mathrm{m}$ depths), where upper mantle and lower crustal sequences have been exposed. Lonsdale [1989] and Fox and Gallo [1989] described the structural settings. Data on dredged samples from the Garrett transform fault are published by Hébert et al. [1983] and Sinton et al. [1991], and detailed submersible studies (Nautile dives, 1991) on the geology of the sampling sites are given by Hekinian et al. [1992] (Figures 1a-1c). The strike-slip motion in the Garrett transform gave rise to steep, faulted scarps and troughs that are oriented roughly perpendicular $\left(095^{\circ}\right)$ to the adjacent EPR axis. This strike-slip motion was coupled with lithospheric extension which is responsible for the recent intratransform volcanic activity in the Alpha, Beta, and Gamma ridges as well as in other smaller volcanic cones.

Transforms offsetting accreting ridge segments can apparently act as cold barriers perturbing mantle flow and causing 

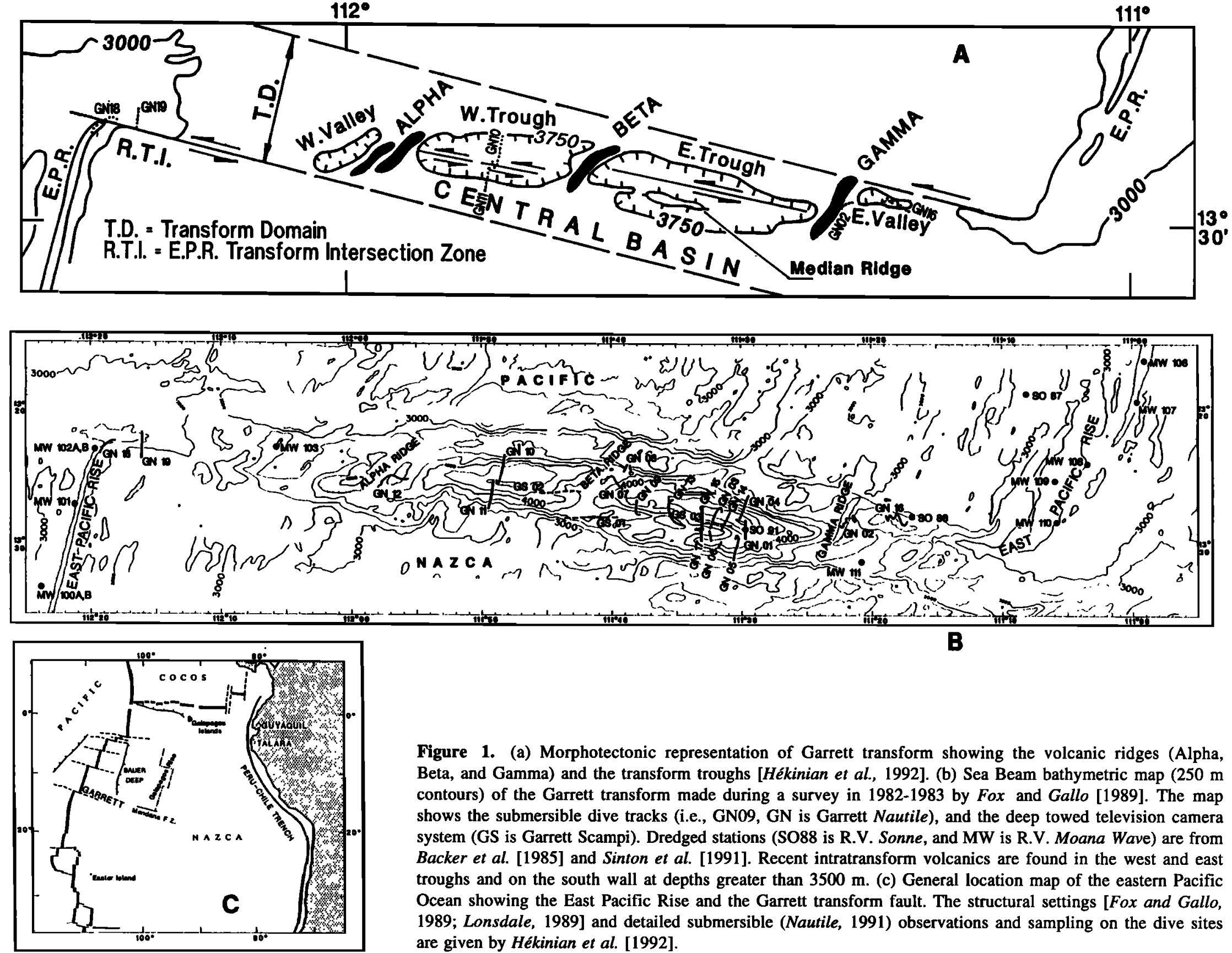

Figure 1. (a) Morphotectonic representation of Garrett transform showing the volcanic ridges (Alpha, Beta, and Gamma) and the transform troughs [Hékinian et al., 1992]. (b) Sea Beam bathymetric map (250 m contours) of the Garrett transform made during a survey in 1982-1983 by Fox and Gallo [1989]. The map shows the submersible dive tracks (i.e., GN09, GN is Garrett Nautile), and the deep towed television camera system (GS is Garrett Scampi). Dredged stations (SO88 is R.V. Sonne, and MW is R.V. Moana Wave) are from Backer et al. [1985] and Sinton et al. [1991]. Recent intratransform volcanics are found in the west and east troughs and on the south wall at depths greater than $3500 \mathrm{~m}$. (c) General location map of the eastern Pacific Ocean showing the East Pacific Rise and the Garrett transform fault. The structural settings [Fox and Gallo, 1989; Lonsdale, 1989] and detailed submersible (Nautile, 1991) observations and sampling on the dive sites are given by Hékinian et al. [1992]. 
"edge effects" on mid-oceanic ridge basalt (MORB) compositions [Bender et al., 1984; Batiza and Niu, 1992]. Transforms may also represent separate zones of magmatic upwelling, or intratransform volcanism, which is different from that of neighboring segments. We show that the presence of several magmatic lineages within the Garrett transform was the result of the fractional crystallization and partial melting of heterogeneous mantle sources. A comparison of the Garrett volcanics with that of other oceanic spreading centers better defines the magmatic processes taking place in various geological settings of the transform.

\section{Analytical Procedures}

The bulk rock analyses including the rare earth element (REE) and trace elements were done by inductively coupled plasma mass spectrometry (ICP-MS) at the Centre de Recherche Petrographique et Geochimique (CRPG) de Nancy, France [Govindaraju, 1989] (Table 1). The electron microprobe analyses on basaltic glass and minerals were obtained using a Camebax SX50 (Microsonde de l'Ouest at IFREMER(Institut Français de Recherche pour l'Exploitation de la MER)) in conjunction with a standard correction program for oxides and silicates (Table 2). The operating conditions were $15 \mathrm{kV}$ accelerating potential, $15 \mathrm{nA}$ specimen current, and $6 \mathrm{~s} / \mathrm{cycle}$ counting time; the beam was focused on a 1-3 $\mu \mathrm{m}$ spot size. The operating conditions for $\mathrm{K}$ and $\mathrm{Ti}$ were extended to $80 \mathrm{nA}$ and $25 \mathrm{~s} /$ cycle for better accuracy. Each individual glassy sample includes on average 3-8 different spots analyzed (Table 2). Analytical precision of the microprobe data based on the average of 25 analyses (Cy82-9-3) is given in Table 3 . The interlaboratory comparison of bulk rock analyses performed on an enriched MORB (Cy82-9-3) is also shown in Table 3.

\section{Intratransform and EPR Axial Volcanics}

\section{Geological Settings}

The Garrett transform fault area is composed of three NESW trending ridges referred to as the Alpha, Beta, and Gamma ridges which obliquely crosscut the main E-W transform trend (Figure 1b). The three oblique ridges are approximately the same size (10 km long and $9 \mathrm{~km}$ wide) and are the sites of the most extensive intratransform volcanic activity [Hékinian et al., 1992]. On the basis of on-site observations in the Garret transform, it is estimated that more than $25 \%$ of the explored area is composed products of recent volcanism. Although the freshest flows are encountered on the oblique ridges (Alpha, Beta, and Gamma), other less prominent recent lava fields forming small mounds (>10 m high) within the eastern trough (samples 9-1, 9-2, and 13-1) and the western trough (sample 10-1) were also observed and sampled at more than 4303$4350 \mathrm{~m}$ depths (Figures 1a, 1b, 2a, and 2d). A recent field of lobated lava invading a small depression called the East Valley bordering the eastern flank of the Gamma ridge was also observed and sampled (16-1 and 16-2) (Figures 1a, 1b, and $2 \mathrm{~b}$ ). Another small lava field was encountered on the south wall of the transform valley (1-12,1-14, and 5-3) at 4615-4718 $\mathrm{m}$ and $3751 \mathrm{~m}$ depth, respectively (Table 1). Small fields of hackly flows partially burying talus piles formed of large dolerite blocks were seen at sampling sites 112 and 1-14 (Figure 2c). These samples (9-1, 9-2, 13-1, 10-
$1,16-1,16-2,1-12,1-14$, and 5-3) from the troughs and walls are referred to as intratransform volcanics. Except for those samples listed above, other recovered samples were not in place; it is unclear whether they are intratransform volcanics or ancient south East Pacific Rise (SEPR) crust. Field observations were the main criteria used for differentiating between intratransform and SEPR volcanism, and because of the uncertain nature of their original settings they are referred to as undifferentiated volcanics (Figure 3a). These undifferentiated volcanics were recovered from talus piles and sedimented areas such as on the north wall (e.g., samples 10-6 and 10-11) of the Western trough and on the Median Ridge (e.g., samples 9-10, 9-12, 9-13, 13-4 and 14-4) associated with metamorphics and unconsolidated breccia near outcrops of serpentinized slivers (Tables 1 and 2, Figures 1a and 1b). All the young recent lavas sampled on the oblique ridges, the transform troughs, and walls are referred to as the Garrett intratransform volcanics (GITV).

Recent volcanism was also observed on the western part of the Garrett transform near the ridge-transform intersection (RTI) zone at 2600-2800 m depths (dive sites GN 18 and GN 19) (Figure 1a). Both areas contain ferrobasalts, and it is believed that the SEPR tip continues into the transform domain. Except for SEPR volcanics, most Garrett intratransform volcanic activity took place at depths greater than $3500-4500 \mathrm{~m}$ on the oblique ridges, transform walls, and transform valley floor.

\section{Petrology}

The Garrett intratransform volcanics are characterized by the occurrence of porphyritic plagioclase and olivine-enriched basalts. This is in agreement with previous observations of Eissen et al. [1981], Natland [1989], and Fornari et al. [1989], who pointed out that porphyritic lavas along the EPR are mainly confined to transform faults. The samples most enriched in olivine $(>10 \%)$ are the picritic basalts (e.g. 9-2, 10-1, and 5-5). Most other samples vary according to their early formed minerals from highly phyric plagioclase $(>10 \%$ ) basalts (HPPB) to moderately phyric up to subaphyric samples containing variable amounts of olivine phenocrysts $(<5 \%)$.

In order to classify the various types of volcanics from the Garrett transform, a comparison has been made with other geological provinces such as zero-age EPR segments. Previous studies of recent volcanics from various north East Pacific Rise (NEPR) segments between $10^{\circ} \mathrm{N}$ and $13^{\circ} \mathrm{N}$ [Hékinian et al., 1989; Thompson et al., 1985, 1989; Langmuir et al., 1986] indicated the occurrence of several types of MORBs. At $11^{\circ}-13^{\circ} \mathrm{N}$, based on $\mathrm{K}$ and $\mathrm{Ti}$ contents, the glassy volcanics were divided into depleted $(\mathrm{K} / \mathrm{Ti}<0.15)$, transitional $(\mathrm{K} / \mathrm{Ti}=\mathbf{0 . 1 5}-\mathbf{0 . 3 5})$, and enriched $(\mathrm{K} / \mathrm{Ti}>0.35)$ MORBs (Figures $3 a-3 c)$. The $K / T i$ ratios are used because they reflect the magmatic source, and for comparative purposes, values for these ratios are usually available since they are analyzed routinely. The volcanics from the fast and ultrafast accreting segments of the EPR show an extensive range of $\mathrm{K} / \mathrm{Ti}$ variability (<0.03-0.5, with $\mathrm{K}_{2} \mathrm{O}=0.03-0.7 \%$ ) (Tables 1 and 2, Figures $3 \mathrm{~b}$ and $3 \mathrm{c}$ ). All the Garrett samples have low values for their $\mathrm{K} / \mathrm{Ti}$ ratios $(<0.2)$ and $\mathrm{K}_{2} \mathrm{O}$ contents $(<0.25 \%$ ) (Figures $3 \mathrm{a}$ and 3d). The ones that erupted in the transform itself consist of depleted MORBs with low $\mathrm{K}_{2} \mathrm{O}$ contents $(0.02-0.11 \%), \mathrm{Ba}$ (<10 ppm), $\mathrm{Zr}(<100 \mathrm{ppm}$ ), and K/Ti (<0.1) (Figure 3a). Both 
Table 1. Bulk Rock Analyses of Extrusives From the Garrett Transform Fault on the EPR Near $13^{\circ} 27 \mathrm{~S}$

\begin{tabular}{|c|c|c|c|c|c|c|c|c|c|c|}
\hline \multicolumn{11}{|c|}{ Sample } \\
\hline & GN2-2 & GN2-7 & GN2-8 & GN7-1 & GN7-7 & GN7-8 & GN08-01 & GN12-4 & GN12-8 & GN12-9 \\
\hline $\begin{array}{c}\text { Bulk Rock } \\
\text { Province }\end{array}$ & $\begin{array}{r}\text { Basalt } \\
\text { Gamma } \\
\text { Ridge }\end{array}$ & $\begin{array}{r}\text { Basalt } \\
\text { Gamma } \\
\text { Ridge }\end{array}$ & $\begin{array}{r}\text { Basalt } \\
\text { Gamma } \\
\text { Ridge }\end{array}$ & $\begin{array}{r}\text { Basalt } \\
\text { Beta } \\
\text { Ridge }\end{array}$ & $\begin{array}{r}\text { Basalt } \\
\text { Beta } \\
\text { Ridge }\end{array}$ & $\begin{array}{r}\text { Basalt } \\
\text { Beta } \\
\text { Ridge }\end{array}$ & $\begin{array}{r}\text { Basalt } \\
\text { Beta } \\
\text { Ridge }\end{array}$ & $\begin{array}{l}\text { Basalt } \\
\text { Alpha } \\
\text { Ridge }\end{array}$ & $\begin{array}{l}\text { Basalt } \\
\text { Alpha } \\
\text { Ridge }\end{array}$ & $\begin{array}{l}\text { Basalt } \\
\text { Alpha } \\
\text { Ridge }\end{array}$ \\
\hline $\begin{array}{l}\mathrm{SiO}_{2} \\
\mathrm{TiO}_{2}\end{array}$ & $\begin{array}{r}50.12 \\
1.13\end{array}$ & $\begin{array}{r}50.27 \\
1.16\end{array}$ & $\begin{array}{r}50.09 \\
1.13\end{array}$ & $\begin{array}{r}50.17 \\
0.96\end{array}$ & $\begin{array}{r}50.53 \\
0.96\end{array}$ & $\begin{array}{r}50.42 \\
0.96\end{array}$ & $\begin{array}{r}49.97 \\
0.88\end{array}$ & $\begin{array}{r}49.42 \\
1.28\end{array}$ & $\begin{array}{r}49.37 \\
1.06\end{array}$ & $\begin{array}{r}50.01 \\
1.37\end{array}$ \\
\hline $\mathrm{Al}_{2} \mathrm{O}_{3}$ & 15.26 & 14.93 & 15.01 & 15.56 & 15.16 & 15.21 & 16.85 & 16.23 & 16.06 & 16.38 \\
\hline $\mathrm{FeO}$ & 8.40 & 8.65 & 8.59 & 7.76 & 8.13 & 8.21 & 7.31 & 8.08 & 7.91 & 8.32 \\
\hline $\mathrm{Fe}_{2} \mathrm{O}_{3}$ & 1.22 & 1.25 & 1.19 & 1.08 & 1.23 & 1.12 & 0.87 & 1.30 & 1.15 & 1.31 \\
\hline $\mathrm{MnO}$ & 0.16 & 0.17 & 0.17 & 0.15 & 0.16 & 0.17 & 0.10 & 0.15 & 0.15 & 0.17 \\
\hline $\mathrm{MgO}$ & 8.10 & 8.03 & 8.16 & 8.75 & 8.58 & 8.64 & 8.19 & 8.05 & 8.89 & 6.83 \\
\hline $\mathrm{CaO}$ & 12.71 & 12.64 & 12.71 & 12.82 & 12.64 & 12.71 & 13.21 & 12.14 & 12.50 & 12.23 \\
\hline $\mathrm{Na}_{2} \mathrm{O}$ & 2.41 & 2.42 & 2.41 & 2.24 & 2.15 & 2.16 & 2.02 & 2.52 & 2.37 & 2.75 \\
\hline $\mathrm{K}_{2} \mathrm{O}$ & 0.05 & 0.05 & 0.05 & 0.04 & 0.03 & 0.03 & 0.05 & 0.06 & 0.05 & 0.06 \\
\hline $\mathrm{P}_{2} \mathrm{O}_{5}$ & 0.10 & 0.10 & 0.10 & 0.08 & 0.08 & 0.08 & 0.10 & 0.13 & 0.11 & 0.15 \\
\hline LOI & -0.61 & -0.65 & -0.66 & -0.51 & -0.56 & -0.68 & -0.54 & -0.30 & -0.48 & -0.67 \\
\hline Total & 99.05 & 99.02 & 98.95 & 99.10 & 99.08 & 99.03 & 99.01 & 99.06 & 99.14 & 98.92 \\
\hline \multicolumn{11}{|c|}{ Trace Elements, ppm } \\
\hline $\mathrm{Ba}$ & 2.55 & 2.32 & 2.53 & 2.18 & 1.58 & 1.71 & 2.43 & 2.95 & 2.7 & 2.96 \\
\hline $\mathrm{Sr}$ & 80 & 78 & 78 & 78 & 47 & 50 & 67 & 86 & 89 & 97 \\
\hline $\mathrm{Nb}$ & 0.7 & 0.4 & 0.3 & 0.3 & $<0.2$ & $<0.2$ & $<5$ & $<0.2$ & $<0.2$ & $<0.2$ \\
\hline $\mathbf{Z r}$ & 56.5 & 55.5 & 56 & 45.5 & 38.8 & 36.7 & 37.7 & 72 & 59 & 76 \\
\hline $\mathbf{V}$ & 301 & 300 & 304 & 250 & 269 & 269 & 258 & 276 & 243 & 327 \\
\hline $\mathbf{Y}$ & 28.4 & 28.5 & 27.1 & 23.3 & 26.3 & 26.6 & 22.5 & 31.5 & 26.9 & 32.6 \\
\hline $\mathrm{Co}$ & 41 & 43 & 41 & 42 & 43 & 41 & 36 & 40 & 43 & 36 \\
\hline $\mathrm{Cr}$ & 393 & 258 & 320 & 378 & 341 & 335 & 424 & 322 & 393 & 194 \\
\hline $\mathrm{Ni}$ & 75 & 71 & 75 & 112 & 110 & 112 & 123 & 129 & 137 & 69 \\
\hline \multicolumn{11}{|c|}{ Rare Earth Elements, ppm } \\
\hline $\mathrm{La}$ & 1.80 & 1.92 & 1.63 & 1.41 & 1.11 & 1.14 & 1.14 & 2.38 & 1.95 & 2.23 \\
\hline $\mathrm{Ce}$ & 8.12 & 6.65 & 5.62 & 5.26 & 4.01 & 4.37 & 3.91 & 7.81 & 6.81 & 7.69 \\
\hline Nd & 6.91 & 6.99 & 6.51 & 5.54 & 5.29 & 5.43 & 3.91 & 8.07 & 7.12 & 7.34 \\
\hline $\mathrm{Sm}$ & 3.19 & 3.1 & 2.95 & 2.64 & 2.66 & 2.79 & 2.07 & 3.72 & 3.11 & 3.25 \\
\hline $\mathrm{Eu}$ & 1.02 & 1.04 & 1.01 & 0.85 & 0.91 & 0.89 & 0.76 & 1.15 & 1.00 & 1.15 \\
\hline Gd & 4.28 & 4.46 & 3.89 & 3.43 & 3.77 & 3.73 & 2.77 & 4.93 & 4.04 & 4.08 \\
\hline Dy & 4.53 & 4.67 & 4.44 & 3.85 & 4.32 & 4.37 & 3.57 & 5.16 & 4.37 & 5.18 \\
\hline $\mathrm{Er}$ & 2.60 & 2.68 & 2.50 & 2.14 & 2.31 & 2.48 & 1.94 & 2.86 & 2.51 & 2.82 \\
\hline $\mathrm{Yb}$ & 2.68 & 2.69 & 2.60 & 2.19 & 2.52 & 2.53 & 2.20 & 2.95 & 2.49 & 2.99 \\
\hline Lu & 0.38 & 0.39 & 0.35 & 0.31 & 0.35 & 0.37 & 0.31 & 0.43 & 0.36 & 0.42 \\
\hline $\mathrm{K} / \mathrm{Ti}$ & 0.061 & 0.058 & 0.061 & 0.061 & 0.04 & 0.042 & 0.074 & 0.067 & 0.066 & 0.063 \\
\hline $\mathrm{Mg} \#$ & 0.66 & 0.65 & 0.65 & 0.69 & 0.68 & 0.68 & 0.69 & 0.66 & 0.69 & 0.62 \\
\hline $\mathrm{Z} \mathbf{r} / \mathrm{Y}$ & 1.99 & 1.95 & 2.07 & 1.95 & 1.48 & 1.38 & 1.68 & 2.29 & 2.19 & 2.33 \\
\hline$(\mathrm{La} / \mathrm{Sm})_{N}$ & 0.36 & 0.40 & 0.36 & 0.28 & 0.27 & 0.26 & 0.36 & 0.41 & 0.40 & 0.44 \\
\hline$(\mathrm{Ce} / \mathrm{Yb})_{N}$ & 0.84 & 0.69 & 0.60 & 0.67 & 0.44 & 0.48 & 0.49 & 0.74 & 0.76 & 0.71 \\
\hline Minerals & pl & pl, ol & pl & pl, ol & pl, ol & pl, ol & pl, ol & pl, ol & $\mathrm{pl}, \mathrm{ol}$ & $\mathrm{pl}, \mathrm{ol}$ \\
\hline $\mathrm{SiO}_{2}$ & 49.34 & 49.37 & 50.03 & 49.92 & 48.76 & 47.20 & 47.77 & 47.72 & 50.53 & 49.90 \\
\hline $\mathrm{TiO}_{2}$ & 0.96 & 1.56 & 1.82 & 1.02 & 1.29 & 0.68 & 0.88 & 0.88 & 1.76 & 2.41 \\
\hline $\mathrm{Al}_{2} \mathrm{O}_{3}$ & 16.67 & 14.82 & 14.30 & 15.10 & 15.91 & 14.96 & 16.75 & 17.25 & 13.93 & 13.28 \\
\hline
\end{tabular}


Table 1. (continued)

\begin{tabular}{|c|c|c|c|c|c|c|c|c|c|c|}
\hline \multicolumn{11}{|c|}{ Sample } \\
\hline & GN13-1 & GN13-4 & GN14-4 & GN01-14 & GN10-6 & GN10-1 & GN9-2 & GN5-5 & GN18-2 & GN18-4 \\
\hline $\begin{array}{l}\text { Bulk rock } \\
\text { Province }\end{array}$ & $\begin{array}{r}\text { basalt } \\
\text { East } \\
\text { Trough* }\end{array}$ & $\begin{array}{r}\text { basalt } \\
\text { Median } \\
\text { Ridge }\end{array}$ & $\begin{array}{r}\text { basalt } \\
\text { East } \\
\text { Trough }\end{array}$ & $\begin{array}{l}\text { basalt } \\
\text { South } \\
\text { wall* }\end{array}$ & $\begin{array}{r}\text { basalt } \\
\text { North } \\
\text { wall }\end{array}$ & $\begin{array}{r}\text { basalt } \\
\text { West } \\
\text { Trough* }\end{array}$ & $\begin{array}{r}\text { picrite } \\
\text { East } \\
\text { Trough* }\end{array}$ & $\begin{array}{r}\text { picrite } \\
\text { South } \\
\text { wall }\end{array}$ & $\begin{array}{l}\text { basalt } \\
\text { R.T.I. }\end{array}$ & $\begin{array}{l}\text { basalt } \\
\text { R.T.I. }\end{array}$ \\
\hline $\mathrm{FeO}$ & 7.63 & 8.50 & 10.21 & 7.82 & 7.50 & 7.32 & 7.40 & 7.14 & 10.48 & 11.33 \\
\hline $\mathrm{Fe}_{2} \mathrm{O} 3$ & 1.02 & 2.17 & 1.49 & 1.66 & 1.06 & 1.33 & 0.89 & 1.13 & 1.49 & 2.33 \\
\hline $\mathrm{MnO}$ & 0.14 & 0.17 & 0.20 & 0.19 & 0.16 & 0.14 & 0.14 & 0.14 & 0.20 & 0.22 \\
\hline $\mathrm{MgO}$ & 8.69 & 7.58 & 7.45 & 8.50 & 9.50 & 14.81 & 11.58 & 10.49 & 6.66 & 5.69 \\
\hline $\mathrm{CaO}$ & 12.66 & 11.41 & 11.25 & 12.71 & 11.25 & 10.96 & 11.48 & 11.75 & 11.10 & 10.23 \\
\hline $\mathrm{Na}_{2} \mathrm{O}$ & 2.29 & 2.67 & 2.70 & 2.22 & 2.62 & 1.82 & 2.58 & 2.52 & 2.90 & 3.08 \\
\hline $\mathrm{K}_{2} \mathrm{O}$ & 0.04 & 0.11 & 0.12 & 0.02 & 0.17 & 0.02 & 0.02 & 0.04 & 0.13 & 0.23 \\
\hline $\mathrm{P}_{2} \mathrm{O}_{5}$ & 0.08 & 0.17 & 0.20 & 0.10 & 0.17 & 0.07 & 0.08 & 0.10 & 0.17 & 0.25 \\
\hline LOI & -0.45 & 0.46 & -0.82 & -0.10 & 0.12 & -0.24 & -0.51 & -0.17 & -0.71 & -0.40 \\
\hline Total & 99.07 & 99.05 & 98.94 & 99.78 & 99.70 & 99.68 & 99.05 & 99.76 & 98.64 & 98.55 \\
\hline \multicolumn{11}{|c|}{ Trace Elements, ppm } \\
\hline $\mathrm{Ba}$ & 4.42 & 8.12 & 9.42 & 2.46 & 15.88 & 0.38 & 2.50 & - & 9.39 & 17.39 \\
\hline $\mathrm{Sr}$ & 73 & 97 & 97 & 78 & 179 & 30 & 92 & 102 & 104 & 111 \\
\hline $\mathrm{Nb}$ & $<0.2$ & 0.4 & 1 & $<0.2$ & 1.3 & $<0.2$ & 0.2 & $<1$ & 2.8 & 3.28 \\
\hline $\mathrm{Zr}$ & 47 & 99 & 109 & 52.63 & 88.22 & 21.25 & 43.8 & 51. & 109 & 149 \\
\hline V & 231 & 316 & 402 & 295 & 251 & 210 & 184 & 192 & 412 & 509 \\
\hline $\mathbf{Y}$ & 25.2 & 38.3 & 43.7 & 24.6 & 25.3 & 18.1 & 22.3 & 20.69 & 42.6 & 53.8 \\
\hline Co & 41 & 41 & 40 & 43 & 41 & 57 & 48 & 47 & 40 & 43 \\
\hline $\mathrm{Cr}$ & 376 & 307 & 233 & 386 & 407 & 1443 & 662 & 511 & 81 & 50 \\
\hline $\mathrm{Ni}$ & 143 & 143 & 102 & 73 & 180 & 484 & 315 & 235 & 54 & 39 \\
\hline \multicolumn{11}{|c|}{ Rare Earth Elements, ppm } \\
\hline $\mathrm{La}$ & 1.80 & 3.60 & 4.37 & 2.09 & 4.78 & 1.35 & 1.22 & 1.18 & 4.18 & 6.70 \\
\hline $\mathrm{Ce}$ & 6.84 & 11.88 & 13.60 & 6.09 & 11.12 & 3.58 & 5.81 & 3.57 & 12.72 & 19.21 \\
\hline $\mathrm{Nd}$ & 6.85 & 10.97 & 12.69 & 6.98 & 9.26 & 2.98 & 6.32 & 5.32 & 12.24 & 17.7 \\
\hline $\mathrm{Sm}$ & 3.22 & 4.51 & 5.21 & 2.84 & 3.19 & 1.87 & 2.73 & 2.28 & 4.72 & 6.74 \\
\hline Eu & 0.98 & 1.37 & 1.60 & 0.92 & 1.09 & 0.66 & 0.93 & 0.84 & 1.51 & 2.02 \\
\hline Gd & 4.13 & 5.47 & 6.12 & 3.56 & 3.83 & 2.53 & 3.58 & 2.98 & 6.07 & 8.16 \\
\hline Dy & 4.15 & 6.19 & 7.04 & 4.23 & 4.36 & 3.17 & 3.51 & 3.27 & 6.89 & 9.01 \\
\hline $\mathrm{Er}$ & 2.44 & 3.16 & 4.03 & 2.33 & 2.13 & 1.52 & 2.27 & 1.79 & 3.87 & 4.99 \\
\hline $\mathbf{Y b}$ & 2.33 & 3.55 & 4.04 & 2.39 & 2.39 & 1.70 & 1.92 & 2.02 & 3.88 & 5.00 \\
\hline Lu & 0.35 & 0.51 & 0.57 & 0.35 & 0.31 & 0.23 & 0.28 & 0.36 & 0.57 & 0.73 \\
\hline $\mathbf{K} / \mathbf{T i}$ & 0.063 & 0.100 & 0.089 & 0.027 & 0.183 & 0.041 & 0.025 & 0.063 & 0.102 & 0.132 \\
\hline $\mathbf{M g} \#$ & 0.69 & 0.64 & 0.59 & 0.68 & 0.72 & 0.80 & 0.76 & 0.74 & 0.56 & 0.50 \\
\hline $\mathrm{Zr} / \mathrm{Y}$ & 1.87 & 2.58 & 2.49 & 2.14 & 3.49 & 1.17 & 1.96 & 2.46 & 2.56 & 2.77 \\
\hline$(\mathrm{La} / \mathrm{Sm})_{N}$ & 0.36 & 0.52 & 0.54 & 0.48 & 0.97 & 0.47 & 0.29 & 0.33 & 0.57 & 0.64 \\
\hline$(\mathrm{Ce} / \mathrm{Yb})_{N}$ & 0.82 & 0.93 & 0.94 & 0.71 & 1.29 & 0.58 & 0.84 & 0.49 & 0.91 & 1.07 \\
\hline Minerals & $\mathrm{pl}, \mathrm{ol}$ & pl, ol & ol & $\mathrm{pl}, \mathrm{ol}$ & ol,pl,spn & ol, spn, pl & ol, pl, spn & ol,pl,spn & pl,cpx,ol & $\mathrm{pl}, \mathrm{cpx}$ \\
\hline
\end{tabular}

Dashes indicate not determined. $\mathrm{Mg} \#=\mathrm{Mg}^{2+} / \mathrm{Fe}^{2+}+\mathrm{Mg}^{2+} \cdot$ Minerals are early formed phases set in glassy matrix. Samples 13-1, 1-14, 10-1, and 9-2 are intratransform volcanics from troughs and walls. (RTI ridge-transform intersection. LOI is loss on ignition. Chemical analyses were done at the Centre de Recherche Pétrographique et Géochimique in Nancy, France Mineral abbreviations are pl, plagioclase; ol, olivine; spn, spinel; and cpx, clinopyroxene. A Jobin Yvon JY 70 spectrometer coupled to a plasma source was used. Analytical precision and accuracy are given by Govindaju [1989].

* Young lavas outcrops. 
Table 2. Microprobe Analyses of Basaltic Glass From the Garrett Transform Fault Near $13^{\circ} 26^{\prime} S$

\begin{tabular}{|c|c|c|c|c|c|c|c|c|c|c|c|c|c|c|c|}
\hline & \multicolumn{15}{|c|}{ Sample } \\
\hline & GN01-04 & GN01-11 & GN01-12 & GN01-14 & GN01-16 & GN02-01 & GN02-02 & GN02-03 & GN02-04 & GN02-05 & GN02-06 & GN02-07 & GN02-08 & GN04-01 & GN04-11 \\
\hline$\overline{\text { Rock }}$ & basalt & basalt & basalt & basalt & basalt & basalt & basalt & basalt & basalt & basalt & basalt & basalt & basalt & basalt & basalt \\
\hline \multirow[t]{2}{*}{ Site } & East & East & East & East & East & Gamma & Gamma & Gamma & Gamma & Gamma & Gamma & Gamma & Gamma & East & Median \\
\hline & Trough & Trough & Trough & Trough & Trough & Ridge & Ridge & Ridge & Ridge & Ridge & Ridge & Ridge & Ridge & Trough & Ridge \\
\hline $\mathrm{SiO}_{2}$ & 50.92 & 50.99 & 50.71 & 51.42 & 50.77 & 50.76 & 50.95 & 50.33 & 50.58 & 50.57 & 50.94 & 50.95 & 50.93 & 50.78 & 50.13 \\
\hline $\mathrm{TiO}_{2}$ & 1.20 & 1.17 & 1.20 & 1.18 & 1.24 & 1.31 & 1.21 & 1.21 & 1.21 & 1.18 & 1.22 & 1.20 & 1.20 & 1.25 & 1.11 \\
\hline $\mathrm{Al}_{2} \mathrm{O}_{3}$ & 14.51 & 14.52 & 14.43 & 14.17 & 14.21 & 14.25 & 14.62 & 15.13 & 14.84 & 15.07 & 14.83 & 14.45 & 14.78 & 14.17 & 15.40 \\
\hline $\mathrm{FeO}^{2}{ }^{3}$ & 9.60 & 9.05 & 9.47 & 10.00 & 9.98 & 9.84 & 9.30 & 9.58 & 9.67 & 9.32 & 9.65 & 9.88 & 9.53 & 9.81 & 9.07 \\
\hline $\mathrm{MnO}$ & 0.17 & 0.17 & 0.17 & 0.11 & 0.18 & 0.18 & 0.25 & 0.18 & 0.17 & 0.18 & 0.20 & 0.14 & 0.18 & 0.19 & 0.19 \\
\hline MgO & 8.16 & 8.37 & 8.11 & 8.11 & 7.98 & 7.92 & 8.33 & 7.78 & 8.29 & 8.45 & 8.18 & 7.97 & 8.30 & 8.05 & 8.95 \\
\hline $\mathrm{CaO}$ & 12.47 & 12.77 & 12.38 & 12.53 & 12.36 & 12.23 & 12.41 & 12.05 & 12.26 & 12.23 & 12.22 & 12.42 & 12.20 & 12.29 & 12.24 \\
\hline $\mathrm{Na}_{2} \mathrm{O}$ & 2.24 & 2.30 & 2.34 & 2.23 & 2.11 & 2.48 & 2.32 & 2.44 & 2.51 & 2.41 & 2.39 & 2.40 & 2.41 & 2.23 & 2.18 \\
\hline $\mathrm{K}_{2}^{2} \mathrm{O}$ & 0.05 & 0.03 & 0.04 & 0.06 & 0.03 & 0.04 & 0.03 & 0.05 & 0.05 & 0.04 & 0.04 & 0.04 & 0.04 & 0.04 & 0.03 \\
\hline $\mathrm{P}_{2}^{2} \mathrm{O}_{5}$ & - & - & - & 0.04 & - & - & 0.09 & 0.09 & 0.05 & 0.08 & 0.08 & 0.06 & 0.06 & - & - \\
\hline Total & 99.32 & 99.37 & 98.85 & 99.85 & 98.85 & 98.99 & 99.51 & 98.84 & 99.62 & 99.54 & 99.75 & 99.52 & 99.62 & 98.80 & 99.30 \\
\hline $\mathrm{K} / \mathrm{Ti}$ & 0.055 & 0.036 & 0.051 & 0.067 & 0.032 & 0.042 & 0.039 & 0.053 & 0.053 & 0.049 & 0.049 & 0.05 & 0.045 & 0.041 & 0.036 \\
\hline $\mathrm{Mg}^{\#}$ & 0.63 & 0.65 & 0.63 & 0.62 & 0.61 & 0.61 & 0.64 & 0.62 & 0.63 & 0.64 & 0.63 & 0.62 & 0.63 & 0.62 & 0.66 \\
\hline
\end{tabular}

Sample

GN04-12 GN05-03 GN05-05 GN05-06 GN07-01 GN07-02 GN07-04 GN07-05 GN07-07 GN07-09 GN08-01 GN08-08 GN09-01 GN09-02 GN09-10

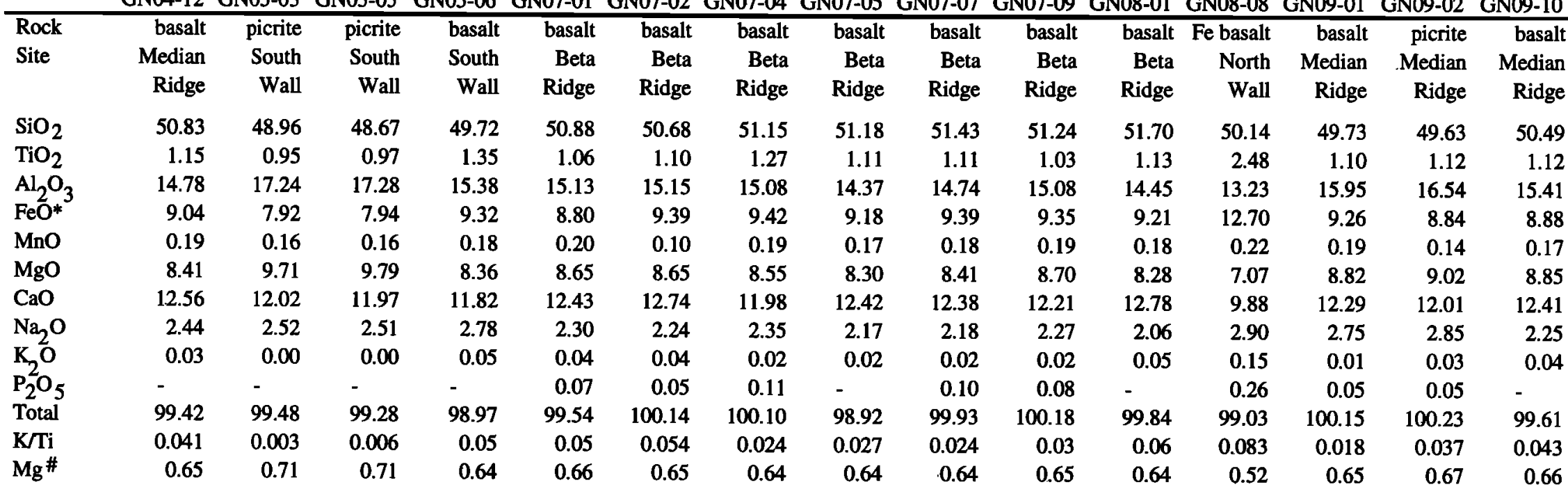


Sample

\begin{tabular}{|c|c|c|c|c|c|c|c|c|c|c|c|c|c|c|c|}
\hline & GN09-12 & GN09-13 & GN10-06 & GN10-11 & GN11-03 & GN11-04 & GN12-01 & GN12-04 & GN12-05. & - GN12-06 & GN12-07 & GN12-08 & GN13-01 & GN13-04 & GN13-06 \\
\hline$\overline{\text { Rock }}$ & basalt & basalt & basalt & Fe basalt & picrite & basalt & basalt & basalt & basalt & basalt & basalt & basalt & basalt & basalt & $\overline{\text { basalt }}$ \\
\hline \multirow[t]{2}{*}{ Site } & Median & Median & West & North & West & West & Alpha & Alpha & Alpha & Alpha & Alpha & Alpha & East & Median & Median \\
\hline & Ridge & Ridge & Trough & Wall & Trough & Trough & Ridge & Ridge & Ridge & Ridge & Ridge & Ridge & Trough & Ridge & Ridge \\
\hline $\mathrm{SiO}_{2}$ & 50.97 & 50.83 & 50.10 & 49.87 & 49.53 & 49.59 & 48.68 & 50.71 & 50.72 & 50.84 & 50.07 & 50.13 & 50.58 & 50.26 & 50.47 \\
\hline $\mathrm{TiO}_{2}$ & 1.25 & 1.24 & 1.49 & 3.27 & 0.98 & 1.28 & 1.35 & 1.51 & 1.59 & 1.76 & 1.28 & 1.09 & 1.16 & 1.68 & 1.73 \\
\hline $\mathrm{Al}_{2} \mathrm{O}_{3}$ & 14.83 & 14.71 & 16.11 & 13.06 & 16.58 & 15.97 & 16.20 & 14.95 & 14.58 & 13.96 & 15.35 & 15.65 & 15.59 & 14.84 & 14.73 \\
\hline $\mathrm{FeO}^{*}{ }^{3}$ & 9.05 & 8.97 & 8.56 & 13.21 & 8.27 & 8.70 & 10.23 & 9.85 & 10.45 & 10.84 & 9.18 & 8.76 & 8.95 & 10.29 & 10.15 \\
\hline $\mathrm{MnO}$ & 0.17 & 0.18 & 0.14 & 0.19 & 0.15 & 0.17 & 0.15 & 0.20 & 0.21 & 0.19 & 0.17 & 0.17 & 0.16 & 0.20 & 0.14 \\
\hline $\mathrm{MgO}$ & 8.47 & 8.35 & 8.82 & 6.28 & 9.35 & 8.90 & 8.16 & 8.04 & 7.64 & 7.19 & 8.53 & 9.09 & 8.79 & 7.78 & 7.57 \\
\hline $\mathrm{CaO}$ & 12.25 & 12.29 & 11.37 & 10.11 & 12.28 & 11.83 & 11.15 & 11.68 & 11.53 & 11.66 & 11.95 & 12.35 & 12.34 & 11.21 & 11.27 \\
\hline $\mathrm{Na}_{2} \mathrm{O}$ & 2.43 & 2.40 & 2.68 & 2.83 & 2.43 & 2.60 & 2.78 & 2.59 & 2.75 & 2.59 & 2.51 & 2.40 & 2.37 & 2.68 & 2.67 \\
\hline $\mathrm{K}_{2} \mathrm{O}$ & 0.04 & 0.04 & 0.24 & 0.18 & 0.00 & 0.03 & 0.04 & 0.06 & 0.07 & 0.05 & 0.05 & 0.03 & 0.04 & 0.09 & 0.11 \\
\hline $\mathrm{P}_{2}^{2} \mathrm{O}_{5}$ & - & - & 0.15 & - & - & - & - & 0.15 & 0.13 & 0.08 & 0.12 & 0.06 & 0.10 & 0.22 & 0.15 \\
\hline Total & 99.45 & 99.00 & 99.65 & 99.01 & 99.58 & 99.07 & 98.73 & 99.74 & 99.67 & 99.16 & 99.20 & 99.72 & 100.09 & 99.23 & 98.99 \\
\hline $\mathrm{K} / \mathrm{Ti}$ & 0.04 & 0.041 & 0.226 & 0.024 & 0.004 & 0.036 & 0.044 & 0.057 & 0.06 & 0.042 & 0.055 & 0.04 & 0.043 & 0.074 & 0.086 \\
\hline $\mathbf{M g} \#$ & 0.65 & 0.65 & 0.67 & 0.49 & 0.69 & 0.67 & 0.61 & 0.62 & 0.59 & 0.57 & 0.65 & 0.67 & 0.66 & 0.60 & 0.60 \\
\hline
\end{tabular}

Sample

\begin{tabular}{|c|c|c|c|c|c|c|c|c|c|c|c|c|c|c|c|}
\hline & GN14-04 & GN15-01 & GN16-01 & GN16-02 & GN16-06 & GN16-07 & GN16-08 & GN18-01 & GN18-02 & GN19-03 & GN19-04 & GN19-05 & GN19-06 & GN19-08 & \\
\hline$\overline{\text { Rock }}$ & basalt & basalt & basalt & basalt & basalt & basalt & basalt & Fe basalt & Fe basalt & Fe basalt & Fe basalt & Fe basalt & Fe basalt & Fe basalt & Fe basalt \\
\hline \multirow[t]{2}{*}{ Site } & Median & Median & North & North & North & North & North & RTI & RTI & RTI & RTI & RTI & RTI & RTI & RTI \\
\hline & Ridge & Ridge & Wall & Wall & Wall & Wall & Wall & RTI & RTI & RTI & RTI & RTI & RTI & RTI & RTI \\
\hline $\mathrm{SiO}_{2}$ & 50.45 & 50.50 & 50.95 & 50.88 & 50.23 & 48.97 & 48.92 & 50.95 & 50.99 & 50.65 & 51.27 & 50.93 & 50.67 & 50.25 & 50.55 \\
\hline $\mathrm{TiO}_{2}$ & 1.88 & 2.45 & 1.31 & 0.95 & 1.18 & 1.34 & 1.30 & 2.08 & 1.87 & 2.95 & 2.81 & 2.05 & 2.74 & 2.63 & 2.50 \\
\hline $\mathrm{Al}_{2} \mathrm{O}_{3}$ & 14.18 & 14.96 & 14.43 & 15.16 & 15.28 & 15.90 & 16.09 & 13.52 & 13.65 & 12.31 & 12.93 & 13.21 & 12.56 & 12.94 & 12.97 \\
\hline $\mathrm{eO}^{*}{ }^{3}$ & 11.27 & 10.07 & 10.05 & 8.43 & 9.20 & 10.29 & 9.76 & 12.34 & 11.90 & 14.54 & 13.94 & 12.46 & 14.21 & 13.82 & 12.74 \\
\hline $\mathrm{MgO}$ & 7.76 & 6.83 & 7.69 & 8.83 & 8.61 & 9.28 & 9.23 & 6.50 & 6.55 & 5.30 & 5.71 & 6.36 & 5.26 & 5.69 & 6.06 \\
\hline $\mathrm{CaO}$ & 10.78 & 10.26 & 12.33 & 13.15 & 12.08 & 10.90 & 10.81 & 10.20 & 10.62 & 9.16 & 9.71 & 10.66 & 9.16 & 9.53 & 9.98 \\
\hline $\mathrm{Na}_{2} \mathrm{O}$ & 2.75 & 3.71 & 2.46 & 2.10 & 2.32 & 2.47 & 2.71 & 3.01 & 2.94 & 3.17 & 1.27 & 2.92 & 3.17 & 3.05 & 2.95 \\
\hline $\mathrm{K}_{2} \mathrm{O}$ & 0.11 & 0.19 & 0.05 & 0.03 & 0.04 & 0.10 & 0.11 & 0.14 & 0.11 & 0.20 & 0.19 & 0.12 & 0.17 & 0.13 & 0.16 \\
\hline $\mathrm{P}_{2}^{2} \mathrm{O}_{5}$ & 0.19 & 0.06 & 0.04 & 0.11 & - & 0.09 & - & - & 0.07 & 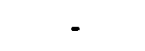 & - & - & - & - & - \\
\hline Total & 99.57 & 99.18 & 99.52 & 99.86 & 99.13 & 99.48 & 99.10 & 98.96 & 98.92 & 98.54 & 98.02 & 98.93 & 98.20 & 98.28 & 98.15 \\
\hline $\mathrm{K} / \mathrm{Ti}$ & 0.08 & 0.106 & 0.057 & 0.05 & 0.041 & 0.099 & 0.12 & 0.092 & 0.085 & 0.095 & 0.093 & 0.084 & 0.086 & 0.069 & 0.091 \\
\hline $\mathrm{Mg}^{\#}$ & 0.58 & 0.57 & 0.60 & 0.67 & 0.65 & 0.64 & 0.65 & 0.51 & 0.52 & 0.42 & 0.45 & 0.50 & 0.42 & 0.45 & 0.49 \\
\hline
\end{tabular}

Dashes indicate not determined. Fe basalt is ferrobasalt (Fe-Ti enriched). Volcanics from Gamma, Beta, and Alpha ridges were erupted in the transform domain. RTI is East Pacific Rise ridge-transform intersection. Samples 1-12, 1-14, 9-1, 9-2, 13-1, 16-1, and 16-2 are young intratransform volcanics from troughs and wall (>3500m

depths). The other trough and wall samples are not in place; they are either intratransform or ancient south EPR volcanics, and they are referred to as "undifferentiated". 
Table 3. Bulk Rock Analyses of Basalts Recovered by the Submersible Cyana in 1984 (Cy84-Prefix) on the Axis of the South East Pacific Rise

\begin{tabular}{|c|c|c|c|c|c|c|c|c|c|c|c|}
\hline & \multirow{2}{*}{$\begin{array}{c}\text { Cy84 } \\
5-6 \\
18^{\circ} 31^{\prime} S\end{array}$} & \multirow{2}{*}{$\begin{array}{c}\text { Cy84 } \\
5-8 \\
18^{\circ} 31 ' S\end{array}$} & \multirow{2}{*}{$\begin{array}{c}\text { Cy84 } \\
5-10 \\
18^{\circ} 31^{\prime} S\end{array}$} & \multirow{2}{*}{$\begin{array}{c}\text { Cy84 } \\
3-1 \\
21^{\circ} 30^{\prime} S\end{array}$} & \multirow{2}{*}{$\begin{array}{c}\text { Cy84 } \\
3-8 \\
21^{\circ} 30^{\prime} \mathrm{S}\end{array}$} & \multirow{2}{*}{$\begin{array}{c}\text { Cy84 } \\
6-2 \\
17^{\circ} 26 ' S\end{array}$} & \multirow{2}{*}{$\begin{array}{c}\text { Cy } 84 \\
7-3 \\
17^{\circ} 27^{\prime} S\end{array}$} & \multicolumn{4}{|c|}{$\begin{array}{c}\text { Cy82 } \\
9-3^{*}\end{array}$} \\
\hline & & & & & & & & $\begin{array}{c}\text { Average } \\
25 \\
\end{array}$ & s.d. & Bulk 1 & Bulk 2 \\
\hline $\begin{array}{l}\mathrm{SiO}_{2} \\
\mathrm{TiO}_{2}\end{array}$ & $\begin{array}{r}50.12 \\
1.62\end{array}$ & $\begin{array}{r}49.27 \\
2.00\end{array}$ & $\begin{array}{r}50.31 \\
1.62\end{array}$ & $\begin{array}{r}50.65 \\
1.41\end{array}$ & $\begin{array}{r}49.97 \\
1.63\end{array}$ & $\begin{array}{r}49.71 \\
1.45\end{array}$ & $\begin{array}{r}49.90 \\
1.52\end{array}$ & $\begin{array}{r}50.32 \\
1.80\end{array}$ & $\begin{array}{l}0.33 \\
0.06\end{array}$ & $\begin{array}{r}50.01 \\
1.84\end{array}$ & $\begin{array}{r}49.29 \\
1.73\end{array}$ \\
\hline $\mathrm{Al}_{2} \mathrm{O}_{3}$ & 14.46 & 13.86 & 14.55 & 14.96 & 14.51 & 14.73 & 14.76 & 16.19 & 0.31 & 16.09 & 16.45 \\
\hline $\mathrm{Fe}_{2} \mathrm{O}_{3}$ & 11.82 & 13.18 & 11.82 & 10.94 & 11.41 & 11.00 & 11.19 & 8.61 & 0.22 & 9.00 & 8.67 \\
\hline $\mathrm{MnO}$ & 0.20 & 0.20 & 0.20 & 0.17 & 0.19 & 0.17 & 0.17 & 0.15 & 0.06 & 0.16 & 0.15 \\
\hline $\mathrm{MgO}$ & 6.84 & 7.00 & 7.00 & 7.66 & 7.40 & 7.75 & 7.65 & 7.51 & 0.21 & 7.54 & 7.47 \\
\hline $\mathrm{CaO}$ & 11.44 & 11.07 & 11.60 & 11.82 & 11.75 & 12.16 & 12.00 & 11.02 & 0.14 & 11.26 & 11.21 \\
\hline $\mathrm{Na}_{2} \mathrm{O}$ & 3.12 & 2.97 & 3.16 & 2.75 & 2.66 & 2.54 & 2.65 & 3.19 & 0.09 & 2.95 & 3.23 \\
\hline $\mathrm{K}_{2} \mathrm{O}$ & 0.12 & 0.05 & 0.08 & 0.06 & 0.05 & 0.05 & 0.12 & 0.43 & 0.03 & 0.39 & 0.42 \\
\hline $\mathrm{P}_{2} \mathrm{O}_{5}$ & 0.19 & 0.20 & 0.19 & 0.15 & 0.17 & 0.16 & 0.17 & 0.24 & 0.06 & 0.22 & 0.28 \\
\hline LOI & -0.25 & -0.77 & -0.27 & -0.68 & -0.62 & -0.61 & -0.68 & - & - & - & - \\
\hline Total & 99.78 & 99.03 & 100.26 & 99.83 & 99.12 & 99.11 & 99.33 & 99.61 & - & 99.46 & 98.90 \\
\hline \multicolumn{12}{|c|}{ Trace Elements, ppm } \\
\hline $\mathbf{B a}$ & 7 & 5 & 5 & - & 9 & 9 & - & - & - & - & - \\
\hline Sr & 136 & 116 & 137 & 100 & 109 & 113 & 121 & - & - & 231 & 231 \\
\hline $\mathrm{Nb}$ & 2 & 2 & 2 & 2 & 1 & 1 & 2 & - & - & 11 & 9 \\
\hline $\mathbf{Z r}$ & 103 & 123 & 103 & 81 & 92 & 84 & 91 & - & - & 163 & 156 \\
\hline V & 323 & 380 & 321 & 314 & 376 & 344 & 339 & - & - & 37 & 31 \\
\hline $\mathbf{Y}$ & 37 & 45 & 35 & 33 & 33 & 30 & 32 & - & - & 255 & 266 \\
\hline Co & 38 & 40 & 37 & 41 & 39 & 40 & 40 & - & - & 39 & 34 \\
\hline $\mathrm{Cr}$ & 108 & 212 & 111 & 276 & 279 & 352 & 309 & - & - & 251 & 268 \\
\hline $\mathrm{Ni}$ & 52 & 73 & 60 & 90 & 79 & 86 & 88 & - & - & 99 & 96 \\
\hline \multicolumn{12}{|c|}{ Rare Earth Eelements, ppm } \\
\hline $\mathbf{L a}$ & 3.43 & 3.92 & 3.46 & 2.44 & 2.94 & 3.53 & 3.50 & - & - & 8.90 & 8.60 \\
\hline $\mathrm{Ce}$ & 11.73 & 13.24 & 11.28 & 10.89 & 10.95 & 10.79 & 10.54 & - & - & 21.00 & 22.50 \\
\hline Nd & 11.04 & 12.32 & 10.45 & 8.22 & 8.66 & 9.59 & 9.21 & - & - & 17.00 & 15.10 \\
\hline $\mathrm{Sm}$ & 4.09 & 4.91 & 3.78 & 3.54 & 3.37 & 3.73 & 2.68 & - & - & 4.10 & 4.59 \\
\hline Eu & 1.33 & 1.64 & 1.38 & 1.24 & 1.19 & 1.22 & 1.26 & - & - & 1.40 & 1.61 \\
\hline Gd & 4.58 & 5.19 & 4.18 & 4.35 & 4.05 & 4.31 & 4.18 & - & - & - & 4.85 \\
\hline Dy & 5.42 & 6.95 & 5.37 & 5.28 & 5.45 & 5.10 & 5.06 & - & - & - & 5.39 \\
\hline Er & 3.54 & 4.24 & 3.41 & 2.64 & 2.74 & 2.67 & 2.67 & - & - & - & 3.14 \\
\hline $\mathbf{Y b}$ & 3.78 & 4.49 & 3.65 & 3.17 & 3.20 & 2.90 & 3.04 & - & - & 2.70 & 3.13 \\
\hline $\mathbf{L}$ & 0.62 & 0.69 & 0.58 & 0.51 & 0.40 & 0.38 & 0.54 & - & - & 0.23 & 0.43 \\
\hline $\mathrm{K} / \mathrm{Ti}$ glass & 0.09 & 0.05 & 0.06 & 0.06 & 0.06 & 0.06 & 0.11 & 0.33 & 0.02 & - & - \\
\hline $\begin{array}{l}\text { Mg \# } \\
\text { glass }\end{array}$ & 0.58 & 0.56 & 0.58 & 0.62 & 0.66 & - & 0.62 & 0.63 & 0.01 & - & - \\
\hline $\mathrm{Zr} / \mathrm{Y}$ & 2.79 & 2.73 & 2.90 & 2.81 & 2.80 & 2.43 & 2.83 & - & - & 4.41 & 4.98 \\
\hline$(\mathrm{La} / \mathrm{Sm})_{N}$ & 0.54 & 0.52 & 0.59 & 0.84 & 0.56 & 0.44 & 0.61 & - & - & 1.40 & 1.21 \\
\hline$(\mathrm{Ce} / \mathrm{Yb})_{N}$ & 0.86 & 0.82 & 0.86 & 0.96 & 0.95 & 0.17 & 1.03 & - & - & 2.16 & 2.00 \\
\hline
\end{tabular}

Analyses were done by the inductively coupled plasma mass spectrometry (ICP-MS) method at the Centre de Recherche Petrographique et Geochimique (CRPG) in Nancy, France. The analytical precision and accuracy are given by Govindaraju [1989]. LOI is loss on ignition.

*Cy82-9-3 is a basalt glass (average 25) analyzed with Camebax SX50; its bulk composition was determined by instrumental newton activation analysis (INAA) (bulk 1, Hékinian et al. [1989]) and ICP-MS (bulk 2, CRPG) . 

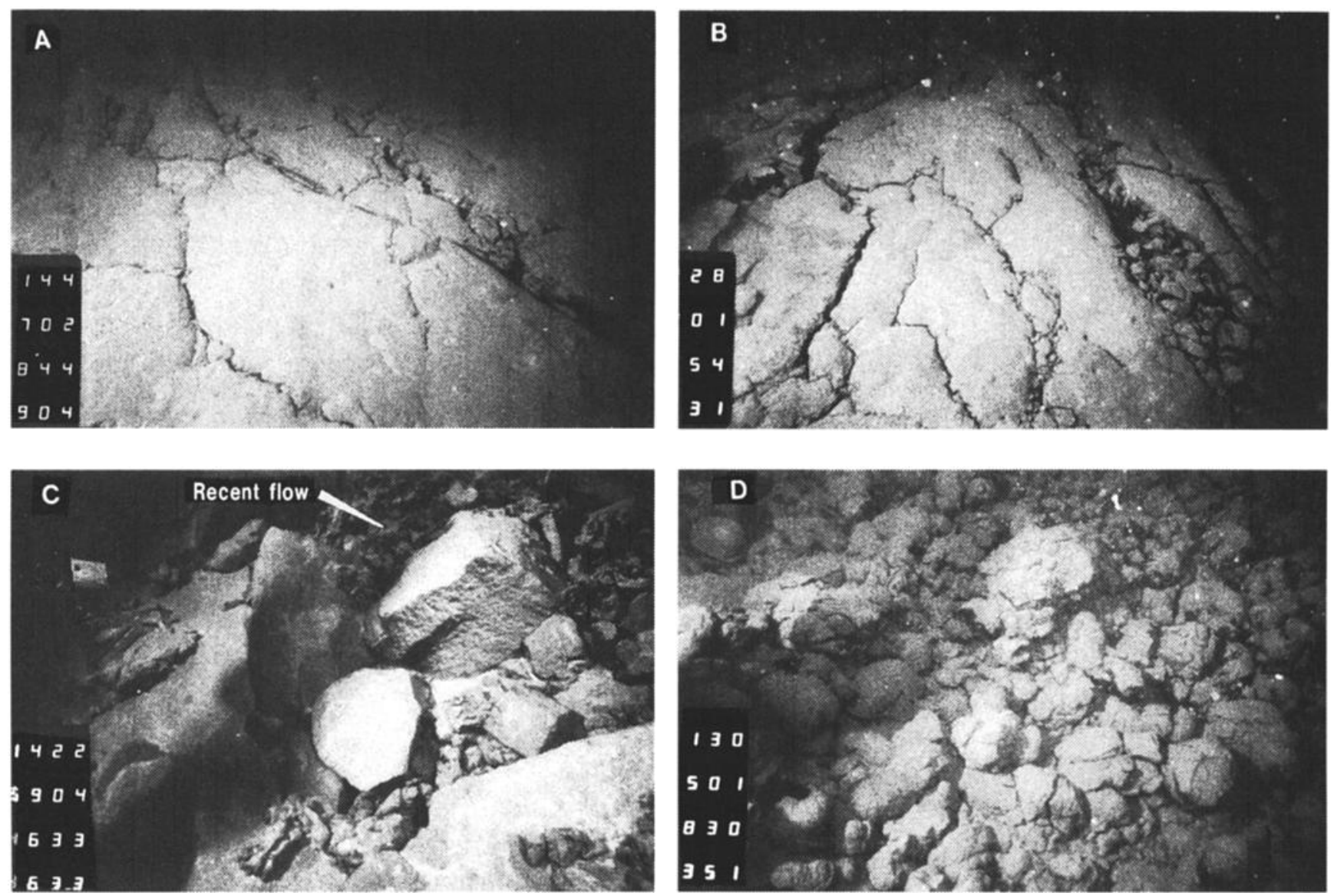

Figure 2. Bottom photographs of intratransform volcanic landscape exposed in the Garrett transform domain. (a) Domed shaped flat flow of basalt from the east trough at dive site GN13 and sample location 13-1 (4528 $\mathrm{m}$ depth). (b) Glassy sheet flow of porphyritic basalt from the East Valley near the eastern flank of the Gamma ridge at dive site GN16 and sample location 16-1 (3910 m depth). (c) South wall overhanging the east trough near longitude $111^{\circ} 30^{\prime} \mathrm{W}$ at dive site GN01 and sample location 1-13 and 1-14 (4627 m depth). Sample 1-14 is a sheet flow partially covering a dolerite at sample site 1-13. (d) Flank of a small volcanic mound of pillow lava formed in the east trough between the northem wall and the Median Ridge (Figures 1a and 1b). Sample 9-2 taken at this site (4350 $\mathrm{m}$ depths) consists of an olivine rich flow.

bulk rock and glass analyses show comparable variation trends which concord with the present classification (Figure 3d). Samples 10-6, 13-4, and 14-4 recovered within the tectonically active transform domain and considered as being ancient SEPR lavas differ from the other Garrett intratransform MORBs by their higher $\mathrm{K} / \mathrm{Ti}(>0.08-0.2), \mathrm{Zr}(\mathbf{8 0}-110$ $\mathrm{ppm})$ and $\mathrm{Ba}(8-16 \mathrm{ppm})$. These three samples are transitional (T-MORB, 10-6) and depleted (D-MORB) basalts, according to the classification of Hékinian et al., [1989] (Figures 3a and 3c). Similarly, the SEPR axial lavas reported here show higher $\mathrm{Zr}$ (>80 ppm), and $\mathrm{Ba}(6-10 \mathrm{ppm})$ than most Garrett samples (Table 3). The other anomalous samples with respect to those from the GITV are the ferrobasalts from the ridgetransform intersection (RTI) zone in the Garrett area (Figure 1c). These ferrobasalts are enriched in $\mathrm{TiO}_{2}(1.7-2.4 \%)$ and in Ba (9-18 ppm) (Tables 1 and 2). A ferrobasalt (sample 8-8) also recovered from the north wall of the transform domain near the intersection with the Beta ridge is believed to be from an ancient RTI zone (Figure 1c).

The Garrett volcanics from the oblique ridges (Alpha, Beta, and Gamma) show moderately depleted chondrite normalized
REE patterns, except for sample 10-6 which has a flatter trend like the T-MORBs from the SEPR at $13^{\circ} 30^{\prime}-21^{\circ} \mathrm{S}$ (Figures 4a-4c). Most of the recent lava flows from the GITV deeper than $3500 \mathrm{~m}$ are among the most depleted in $\mathrm{K}, \mathrm{Ce}$, and $\mathrm{Zr}$ when compared to other SEPR MORBs. The depleted (DMORBs) and transitional (T-MORB) basalts from the SEPR segments show higher $\mathrm{Zr}$ (70-160 ppm), Y (25-60 ppm), and $(\mathrm{La} / \mathrm{Sm})_{N}(0.6-0.9)$ contents than those from the Garrett transform. A few basalts from the Median Ridge (13-4 and 14-4) and from the western trough (10-6) are more enriched in $\mathrm{Zr}$ (88-109 $\mathrm{ppm}),(\mathrm{La} / \mathrm{Sm})_{N}$ (0.6-1), and REE than are the other depleted MORBs from the oblique ridges and walls and troughs (Figures 1b, 4a, 4b, and 4c). These particular Garrett samples (13-4, 14-4, and 10-6) along with the ferrobasalts from the RTI zone are more like the adjacent SEPR volcanics.

\section{Magmatic Processes}

\section{Crystal Fractionation}

Evidence of crystal accumulation is observed in some picritic basalts (10-1, 9-2, and 5-5) where large phenocrysts of 

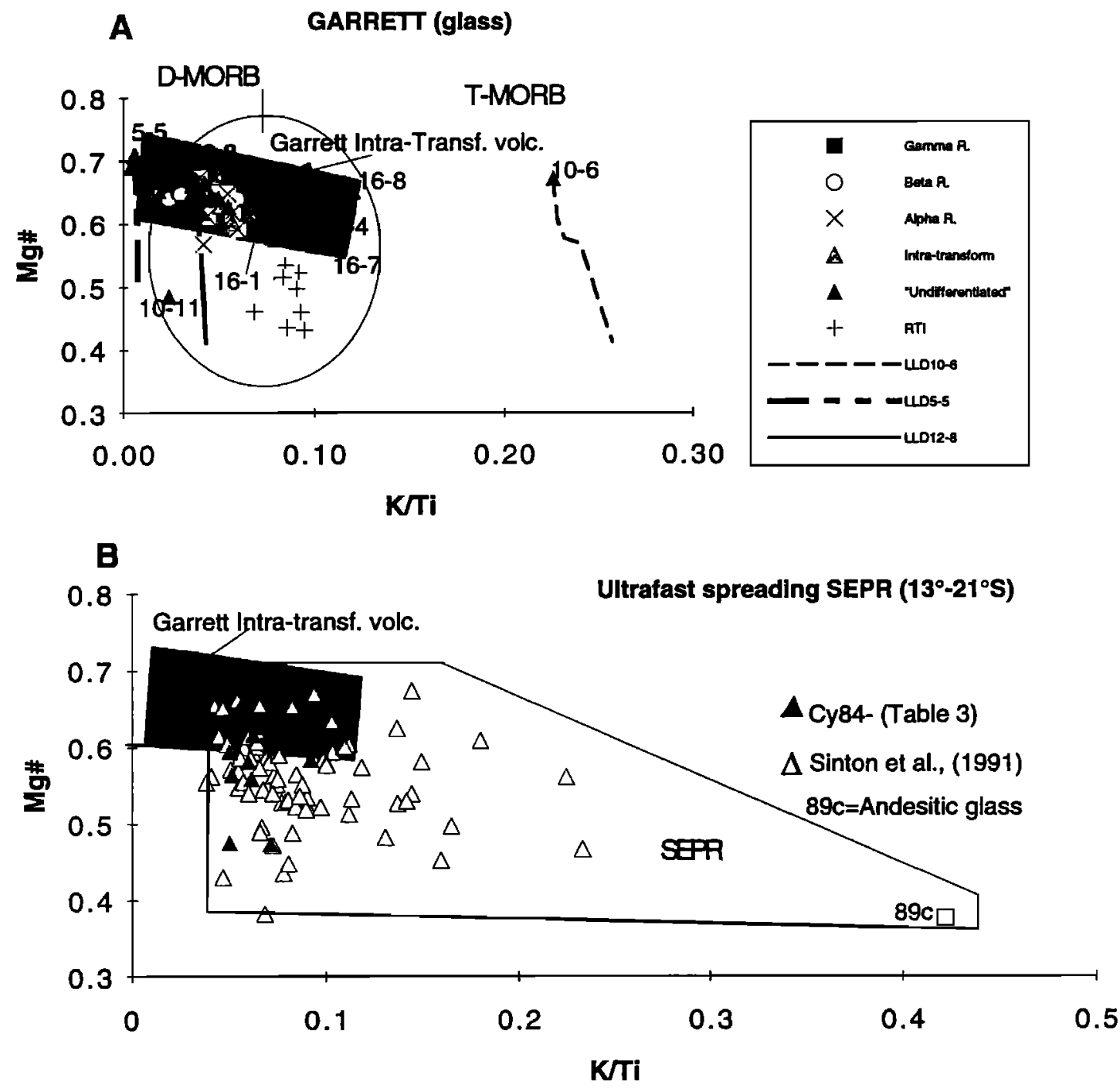

Figure 3. K/Ti-Mg \# variation diagram of glassy chilled margin basalts from various East Pacific Rise segments. (a) The samples from Garrett are collected from the oblique ridges (3000-4000 $\mathrm{m}$ depths) as well as from the transform troughs (>4000 $\mathrm{m}$ depths) and walls (>3300 m depths). Some samples $(5-3,9-2,13-1,1-12,1-$ 14, 16-1, and 16-2) are erupted within the transform domain (intratransform volcanics). The other basalts are either ancient southern East Pacific Rise (SEPR) or intratransform flows whose origins are uncertain (undifferentiated) (see text). Samples from the ridge-transform intersection (RTI) zone consist of ferrobasalts, probably of SEPR origin. Ferrobasalts occur in the transform domain walls (10-11 and 8-8). (b) The glass data (Table 3) consist of samples from the SEPR $\left(13^{\circ}-21^{\circ} \mathrm{S}\right)$ from Sinton et al. [1991] (open triangles) as well as newly analyzed samples (solid triangles, samples Cy84, 17 $26^{\prime}-21^{\circ} 26^{\prime} S$ ) [Renard et al., 1985]. The field (shaded) of the Garrett intratransform volcanics is shown. (c) For comparison with fast spreading segments, selected glass analyses of depleted (D-MORB), transitional (T-MORB) and enriched (E-MORB) from the the EPR axis at $9^{\circ} \mathrm{N}$ (solid diamond) [Allan et al., 1989; Fornari et al., 1988; Batiza and Niu, 1992], 10³0'$11^{\circ} 30^{\prime} \mathrm{N}$ (open diamond) [Hékinian et al., 1989; Thompson et al., 1989] and 13 $\mathrm{N}$ (solid circles) [Hékinian et al., 1989] are reported. Open triangles are off-axial lavas sampled near $13^{\circ} \mathrm{N}$ [Hékinian et al., 1989]. (d) Glass composition compared to bulk rock analyses from Garrett.

olivine and spinel are the early phases which have crystallized in equilibrium with the liquid (Table 1). Occasional compositional zoning (Fo85-89, Fo87-90, and Fo85-88) and the presence of embayed olivine xenocrysts implying nonequilibrium crystallization occur in samples 1-11, 5-5, and 13-4 [Hébert et al., 1994]. In general, however, the positive correlation between the $\mathrm{Mg} \#\left(=\mathrm{Mg}^{+2} / \mathrm{Fe}^{+2}+\mathrm{Mg}^{+2}\right)$ of olivine and glass reflects the progressive fractionation with the increase of $\mathrm{Fe} / \mathrm{Mg}$ (Figure 5a). The importance of fractional crystallization is further supported by the variation trends of very compatible ( $\mathrm{Ni}, \mathrm{Mg} \mathrm{\# )} \mathrm{moderately} \mathrm{compatible}$ (Ca), and incompatible ( $\mathrm{K}$ and $\mathrm{Ti}$ ) elements (Figures $3 \mathrm{a}$, 5a, $5 b, 5 c$, and $6 \mathrm{~b})$. For example, the least evolved basalts (5-5, 10-6, 13-1 and 12-8) have a relatively high bulk rock content of $\mathrm{Ni}$ (140-200 ppm), and high $\mathrm{Mg} \mathrm{\# (0.68-0.71)} \mathrm{glass}$ (Tables 1 and 2, Figure 6a). The least evolved basalts, many 


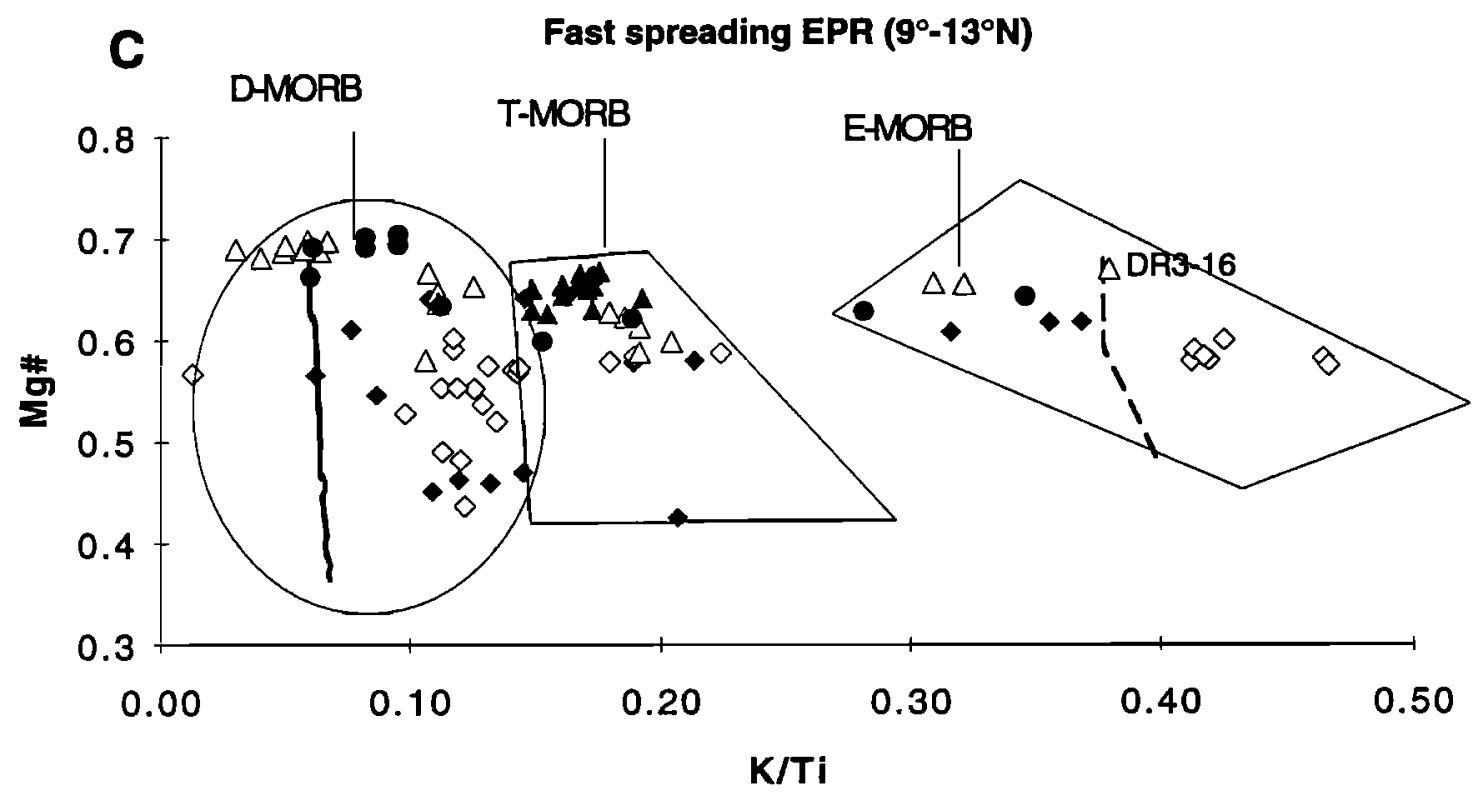

D Glass \& Bulk rock

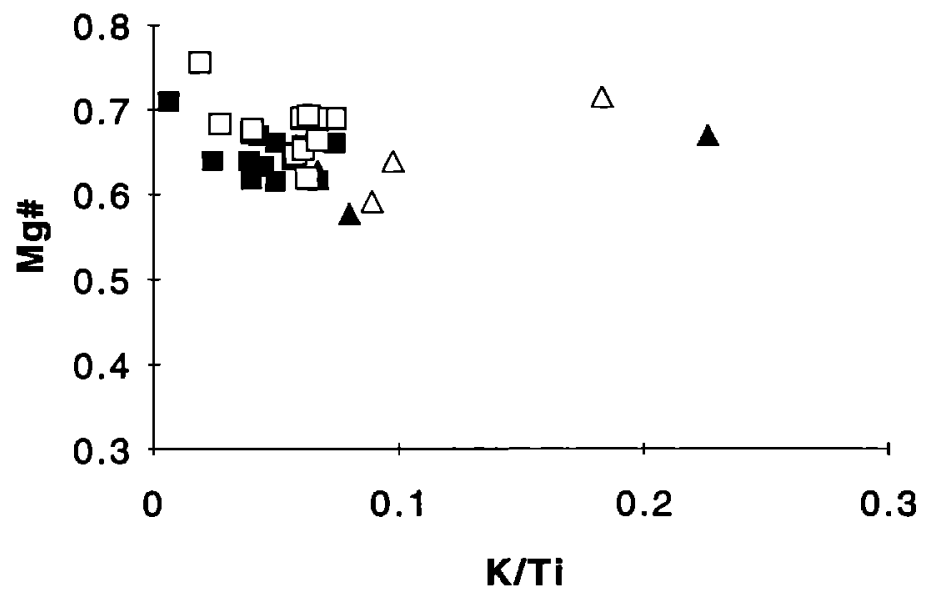

\begin{tabular}{|l|}
\hline Glass,Intra-transform \\
A Glass,Undifferentiated \\
$\square$ Bulk, Intra-transform \\
$\triangle$ Bulk, undifferentiated
\end{tabular}

Figure 3. (continued)

of which are highly porphyritic, are divided into two categories according to their mineral constituents: (1) the porphyritic basalts enriched in plagioclase (5-7\%) and $\mathrm{Mg}$-rich olivine (Fo87-88, 5-7\%) phenocrysts set in a glassy margin and/or cryptocrystalline groundmass (samples 13-1, 12-8) and (2) the olivine basalts containing less than $1 \%$ plagioclase phenocrysts and more than $7 \%$ early formed olivine (Fo89-90) set in a glassy margin and/or intersertial groundmass containing plagioclase and olivine (samples 10-6 and 5-5). Sample 5-5 differs from sample $10-6$ by its lower $\mathrm{K}_{2} \mathrm{O}$ (0.05\%) and $\mathrm{Zr}(51 \mathrm{ppm})$ contents (Table 1$)$.

To evaluate the low-pressure crystal fractionation process, Weaver and Langmuir's [1990] algorithm for the liquid lines of descent (LLD) was used (Figures 3a, 5b, and 5c). Crystal fractionation of less than $30 \%$, and often closer to $20 \%$ (with

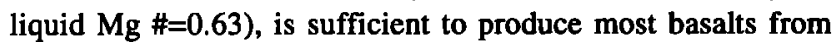
the Garrett transform. The crystal fractionation trends were calculated from initial melts having a composition similar to some of the depleted and least evolved MORBs (e.g. 12-8, 55, 10-6, and 13-1) (Figures 5b-5d). In most cases, spinel, olivine, and olivine + plagioclase are the first phases to crystallize, followed by clinopyroxene which starts to form when the total crystallization reaches around $15-20 \%$, corresponding to a liquid with an $\mathrm{Mg}$ \# (glass) of about 0.63-0.64. Early crystallization of olivine and plagioclase increases the $\mathrm{CaO} / \mathrm{Al}_{2} \mathrm{O}_{3}(0.4-0.7)$ ratio in the liquid $(\mathrm{Mg} \#$ of 0.67-0.68) until attaining the cotectic where clinopyroxene, olivine, and plagioclase crystallize together. This will decrease the $\mathrm{CaO} / \mathrm{Al}_{2} \mathrm{O}_{3}(<0.8)$ at the same time as the $\mathrm{Mg}$ \# values $(<0.64)$ decrease (Figures $5 \mathrm{c}$ and $5 \mathrm{~d}$ ). The samples with the highest $\mathrm{CaO} / \mathrm{Al}_{2} \mathrm{O}_{3}(>0.8)$ values do not fit any pattern of a crystal-liquid line of fractionation as calculated from some of the least evolved melt compositions (Figure 5d). This difference might be inherited from a more depleted $\left(\mathrm{Na}_{2} \mathrm{O}\right)$ parental melt than the other least evolved basalts. Melts containing large amounts of plagioclase would be segregated and abnormally accumulated in restricted conduits. Indeed, the basalts (1-14, 1-16, 2-6, 8-1, 7-5, and 16-2) having high $\mathrm{CaO} / \mathrm{Al}_{2} \mathrm{O}_{3}$ $(>0.85)$ ratios and lower $\mathrm{Mg} \#(<0.65)$, and lower $\mathrm{Na}_{2} \mathrm{O}$ $(<2.5 \%)$ contents contain abundant $(10-15 \%)$ plagioclase 


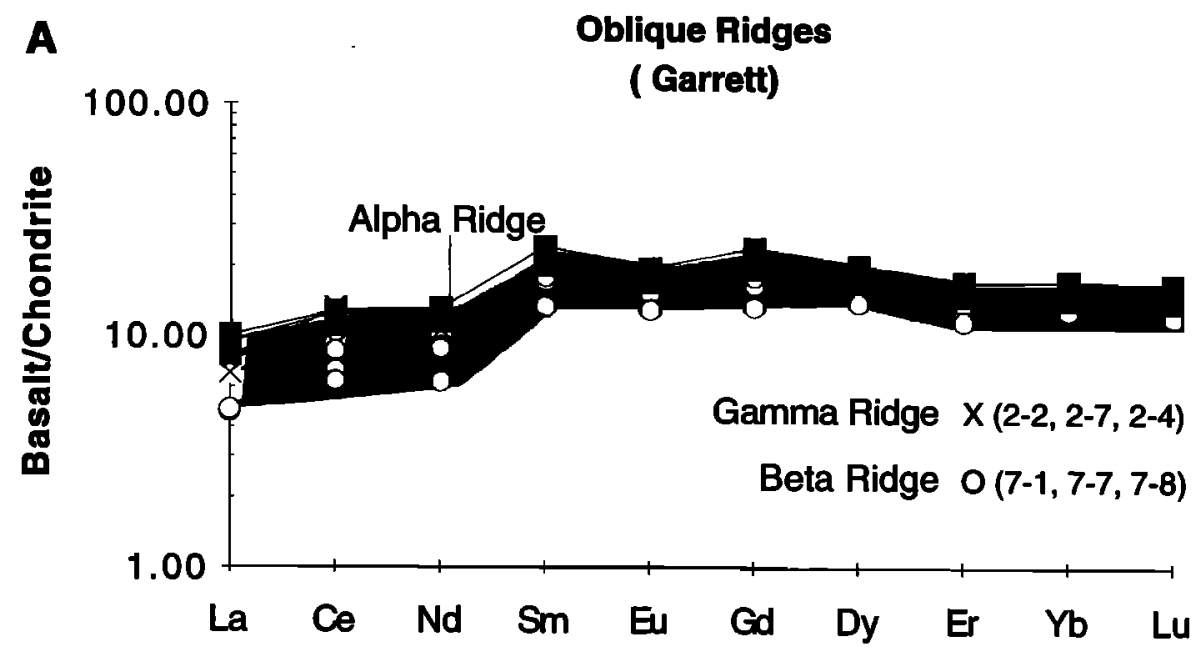

\section{Troughs \& Walls basalts}
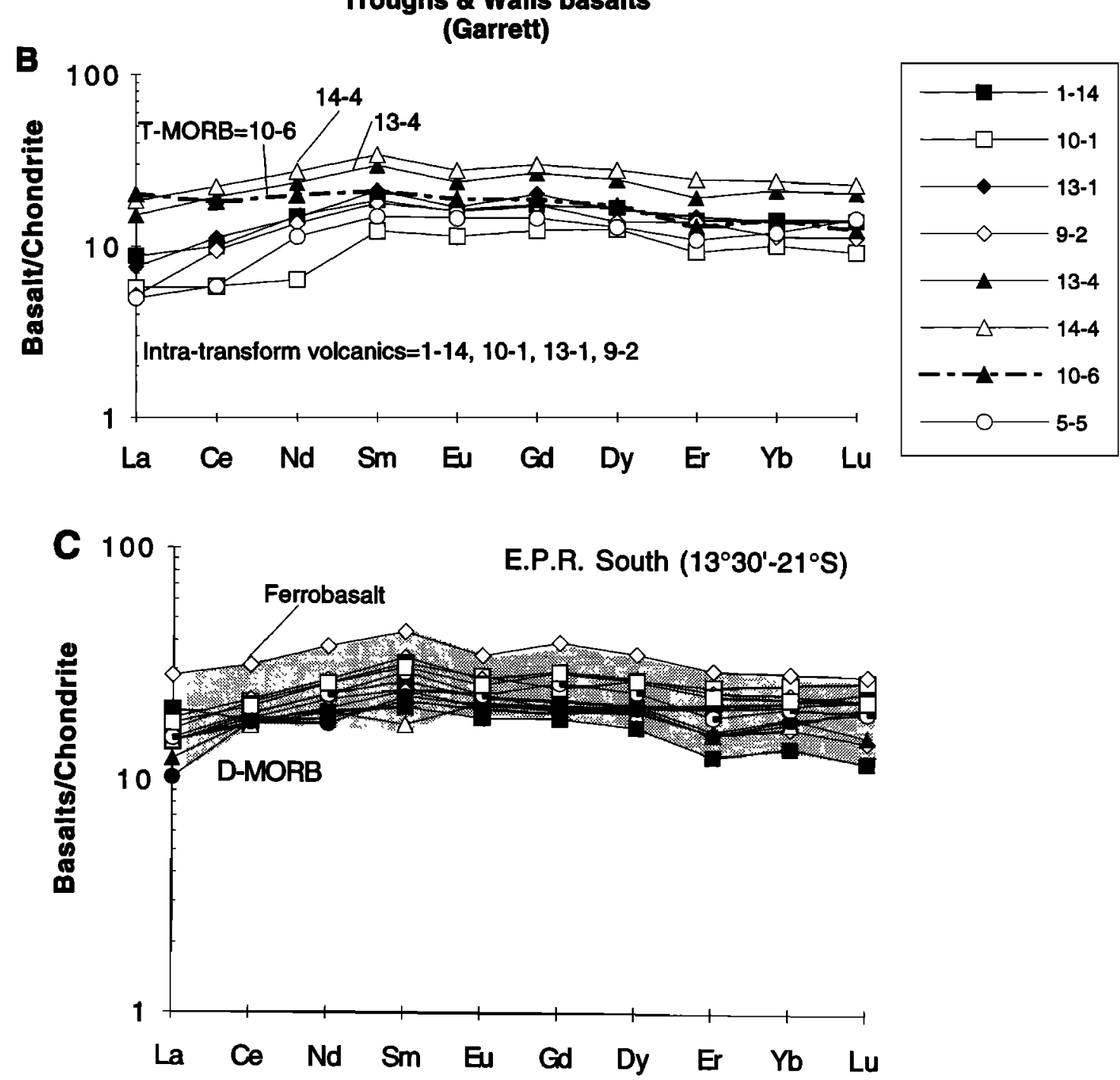

Figure 4. Bulk rare earth elements normalized to chondrite (C1) [Sun and McDonough, 1989] variation diagram of basaltic rocks from the Garrett transform faults. The sample numbers are the same as in Tables 1 and 3. (a) Samples from the oblique ridges (Gamma, crosses; Beta, open circles; and Alpha, solid squares) are shown. (b) Basalts from the eastern (13-1 and 9-2) and western (10-6 and 10-1) troughs, the Median Ridge (14-4 and 13-4), and the walls (5-5 and 1-14) are reported in Table 1. (c) The ferrobasalts (GN18-) from the ridgetransform intersection (RTI) in the Garrett area are compared with the southern East Pacific Rise (SEPR) basalts (Tables 2 and 3). Other SEPR basalts (dotted field) are from Bach et al. [1994] and Mahoney et al. [1994]. 

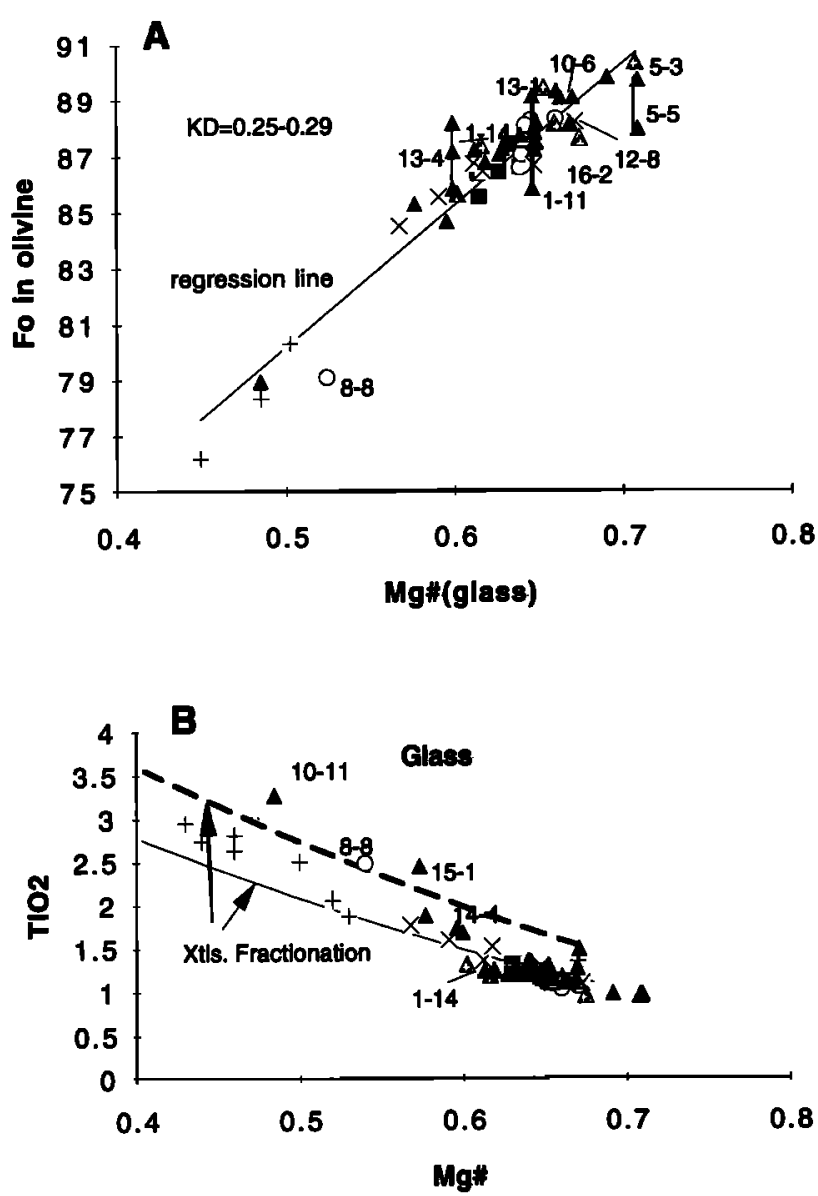
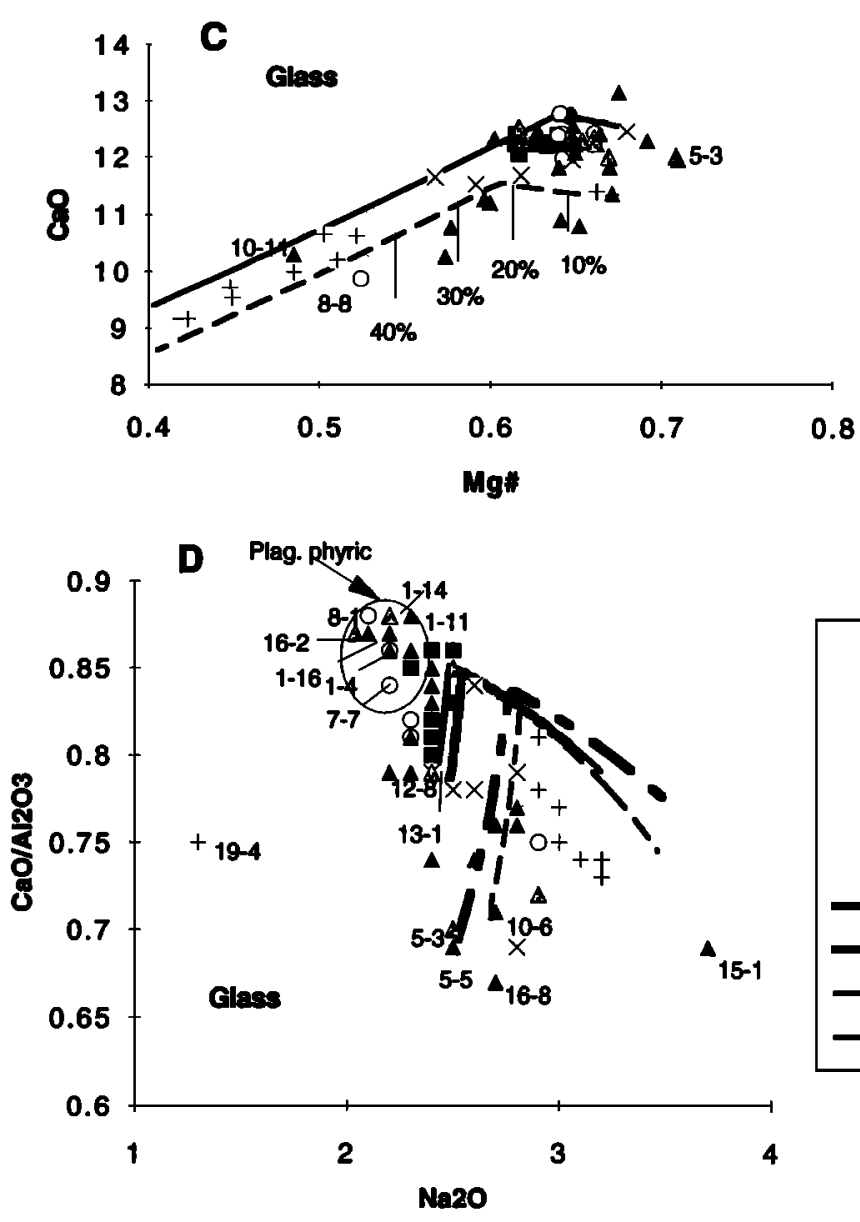

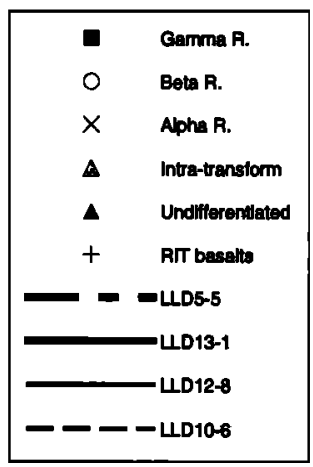

Figure 5. (a) Forsterite (Fo)-Mg \# (total $\mathrm{Fe}$ as $\mathrm{FeO}$ ) variation diagram of olivine and basaltic glass from various geological settings in the Garrett transform and ridge-transform intersection (RTI) zone. The intratransform volcanics refer to samples 5-3, 13-1, 9-1, 9-2, and 1-14. Samples 9-1 and 9-2 are not labeled in Figure. Samples 5-5, 1-11, and 13-4 with large scatter (solid lines) in olivine composition (Fo85-90) consist of xenocrysts and zoned crystals. Sample 8-8 is a ferrobasalt from the north wall of the transform near the Beta Ridge. (b) $\mathrm{Mg}$ \#-TiO 2 variation diagram of basaltic glass showing the two trends of crystal fractionation for the least evolved T-MORB (10-6) and D-MORB (12-8). (c) Mg \#-CaO variation diagram showing the calculated trends of crystal fractionation (liquid line of descent, LLD) with the percent of crystallization. The Garrett samples, except for the RTI ferrobasalts, are produced with less than 30\% crystallization (Mg \# $=0.64-0.57$ ) Most samples average 20\% (Mg \# of 0.62) for depleted MORBs. Symbols are the same as in Figures 3b and 3d. (d) $\mathrm{CaO} / \mathrm{Al}_{2} \mathrm{O}_{3}-\mathrm{Na}_{2} \mathrm{O}$ variation diagram showing the calculated trends of crystal fractionation (LLD). 

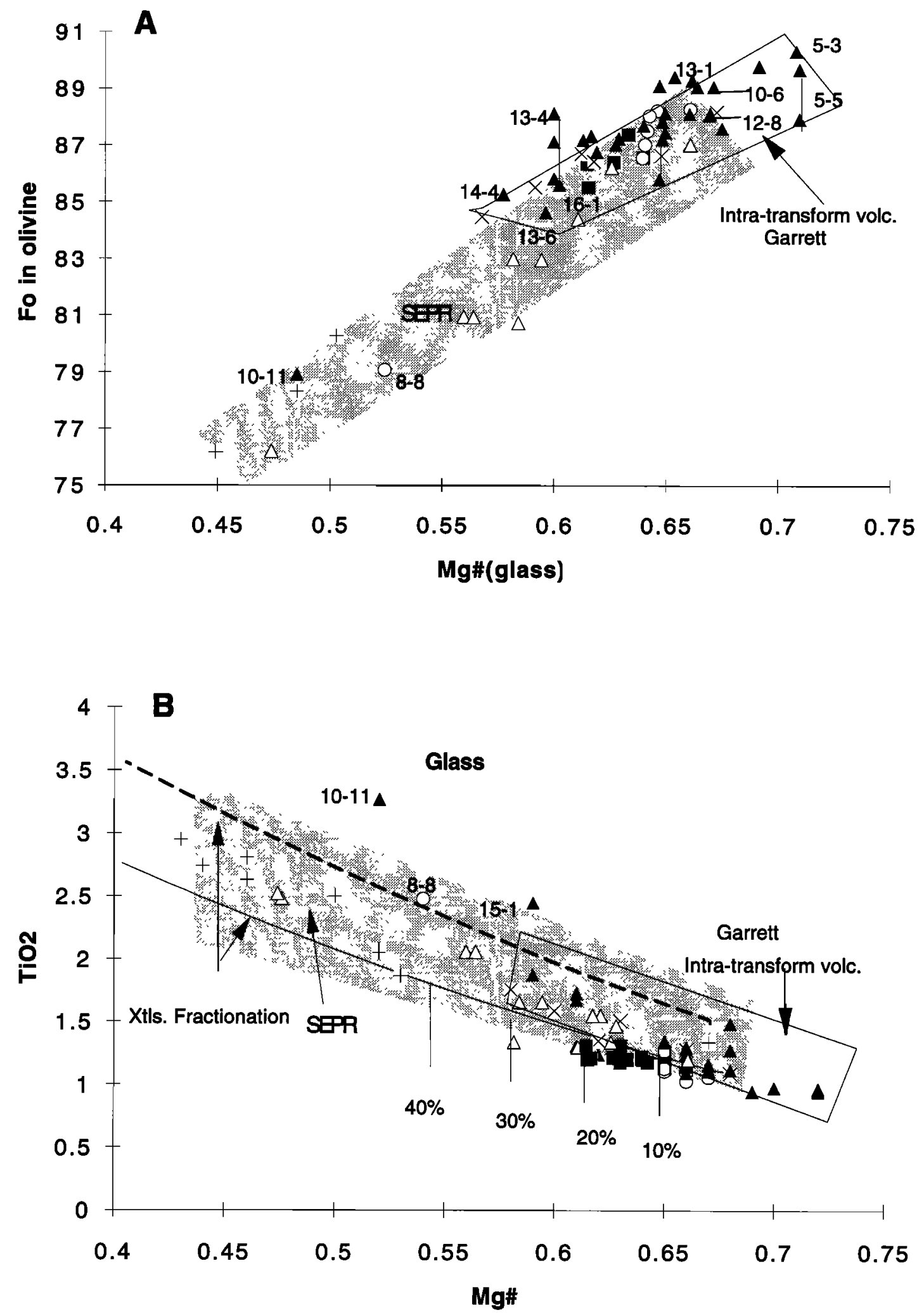

Figure 6. (a) Forsterite (Fo) versus $\mathrm{Mg}$ \# (total $\mathrm{Fe}$ as $\mathrm{FeO}$ ) variation diagram of olivine and basaltic glass (microprobe data) from the Garrett transform and the ridge-transform intersection (RTI) zones compared with the souhern East Pacific Rise (SEPR) (shaded area and empty triangles) volcanics (Cy84-). (b) $\mathrm{Mg \# -TiO}_{2}$ variation diagram of Garrett and other SEPR volcanics. The SEPR field contains samples from Sinton et al . [1991] and from Cyana 1984 (Cy84-) dives (open triangles) (Table 3). Symbols are the same as in Figures 3a, $3 \mathrm{~b}$, and $5 \mathrm{~b}$. 


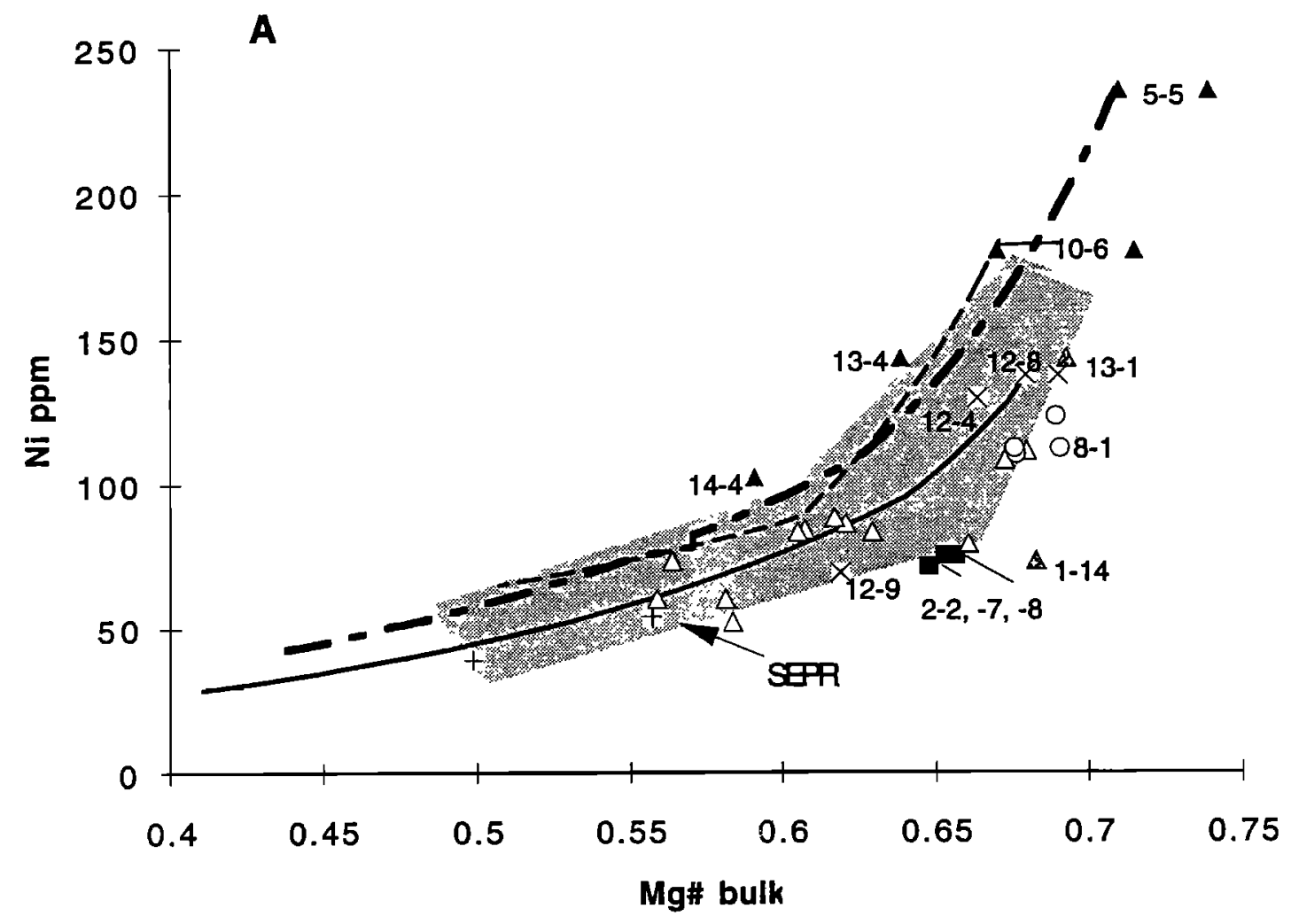

B

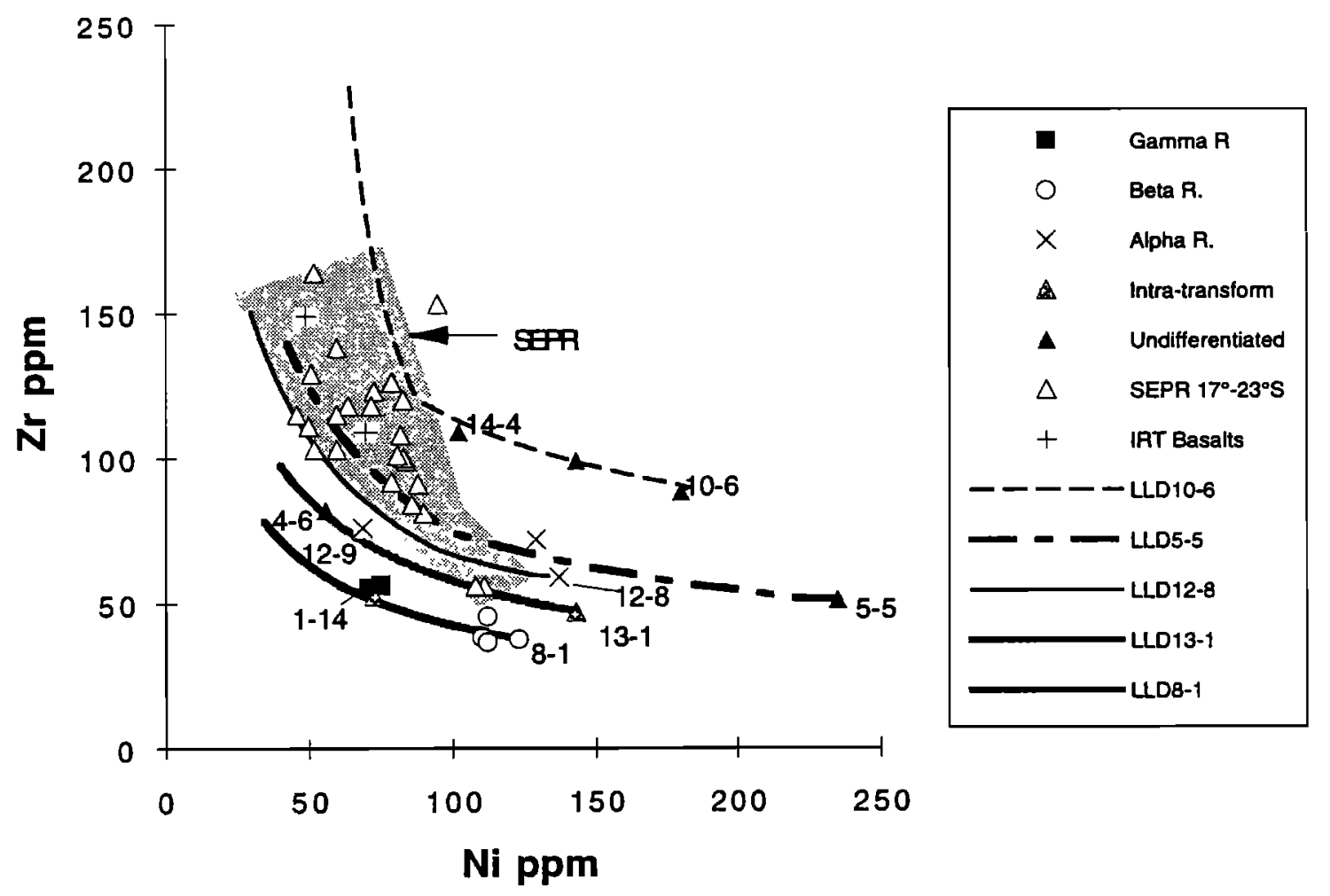

Figure 7. (a) $\mathrm{Ni}-\mathrm{Mg}$ \# and (b) $\mathrm{Zr}-\mathrm{Ni}$ variation diagram of basaltic rocks. The liquid lines of descent (LLD) are calculated from the least evolved glassy basalts $(5-5,10-6,12-8,13-1$ and 8-1). The symbols are the same as in Figure 3a. Bulk chemical analyses from Garrett and southern East Pacific Rise (SEPR) are reported in Tables 1 and 3. Others are unpublished analyses from Cyana 1984 dives and from Bach et al. [1994] and Mahoney et al. [1994]. 
megacrysts and/or phenocrysts (Figure 5d). The plagioclase retains about twice as much $\mathrm{Al}_{2} \mathrm{O}_{3}$ as $\mathrm{CaO}$ which increases the $\mathrm{CaO} / \mathrm{Al}_{2} \mathrm{O}_{3}$ ratios in the glass.

The most evolved lava (samples 18-2 and 18-4) from the RTI are almost aphyric with rare plagioclase $(<1 \%)$ and clinopyroxene phenocrysts and are enriched in total FeO (11$13 \%)$ and $\mathrm{TiO}_{2}(1.8-2.5 \%)$ (Table 1 and Figures $6 \mathrm{a}$ and $6 \mathrm{~b}$ ).

They are comparable to the ferrobasalts found elsewhere on accreting ridges [Clague and Bunch, 1976]. The ferrobasalts are depleted in $\mathrm{K} / \mathrm{Ti}(0.1)$ and fall along the evolved pattern defined by the crystal fractionation model (Figures $3 a, 5 b$, and 5c). Their olivine, when present, is depleted in forsterite (Fo75-77), and they have a low Mg \# (0.45-0.53) (Figure 6b). When the total amount of crystallized phases reaches about $30 \%$, the liquid has a low $\mathrm{Mg} \#(<0.60)$, and the amount of olivine formed in equilibrium has attained its maximum (about 10\%). Further crystallization will give rise to Fe- and Ti-rich liquids as expected. The fact that the RTI lavas fall in a field of lower $\mathrm{CaO} / \mathrm{Al}_{2} \mathrm{O}_{3}$ ratio than the predicted crystal fractionation trends (LLD), suggests that they could have been formed from another more Alkali enriched parental melt (Figure 5d).

The oblique ridges and the intratransform volcanics from Garrett are generally less evolved than the SEPR basalts. They show higher $\mathrm{Mg}$ \# $(>0.58)$, Ni $(100-480 \mathrm{ppm})$ content and forsterite content for their olivine (Fo84-90) than those from the SEPR (Mg \#=0.48-0.70, Ni=40-200 ppm, Fo76-89) (Figures 6a, 6b, 7a, and 7b). However, the samples from the Gamma (2-2, 2-7, and 2-8), and the Alpha (12-9) oblique ridges and one sample from the south wall (1-14) have low $\mathrm{Ni}$ (69-75 ppm) for a relatively high $\mathrm{Mg} \#(0.65-0.68)$. It is likely that these later samples represent a different fractionation lineage derived from a parental melt source more depleted in its $\mathrm{Zr}$ content than the other volcanics from the Garrett transform (Figure 7b). The other more evolved samples consisting of the ferrobasalt (8-8) and aphyric basalts (10-11 and 14-4) with relatively low $\mathrm{Mg} \#(<0.60)$ are believed to have a similar origin to that of the SEPR volcanics (Figures 7a and 7b).

\section{Parental Melt and Melting Process}

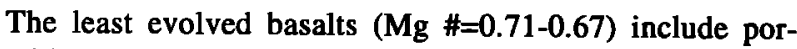
phyritic rocks with euhedral phenocrysts in equilibrium with their liquids (samples 12-8, 13-1, and 10-6). Samples 12-8 and 13-1, which are depleted in incompatible elements, have comparable compositions and show close trends of variabilities for their liquid lines of descent (Figures 5d and 7b). Most intratransform volcanics are found distributed around this fractionation trend, but sample $10-6$ is enriched in incompatible elements $\left(\mathrm{K}_{2} \mathrm{O}=0.17-0.25, \mathrm{Ce}>10 \mathrm{ppm}, \mathrm{Y}>25 \mathrm{ppm}, \mathrm{Zr}>80\right.$ ppm, and $\mathrm{Zr} / \mathrm{Y}>2.5$ ). This sample (10-6) together with samples 13-4 and 14-4 have $\mathrm{Zr} / \mathrm{Y}$ and $\mathrm{Ce} / \mathrm{Yb}$ ratios comparable to the basalts collected from the SEPR $(\mathrm{Zr} / \mathrm{Y}=2.5-3.5$ and $\left.(\mathrm{Ce} / \mathrm{Yb})_{N}=0.8-1.3\right)$ (Figures 8a and $8 \mathrm{~b}$ ). This distinct liquid line of descent as defined by sample 10-6 suggests the coexistence of different parental magmas originated from the melting of heterogeneous mantle (Figures 3a, 5b, 5c, 6a, 6b, 8a, and 8b). At least two parental melts are required: (1) a depleted end-member with low $\mathrm{K} / \mathrm{Ti}(0.04-0.10)$, low $\mathrm{Zr} / \mathrm{Y}(<2)$, $(\mathrm{Ce} / \mathrm{Yb})_{N}<1$, and $(\mathrm{La} / \mathrm{Sm})_{N}<1$, and (2) a more enriched parental melt with higher $\mathrm{K} / \mathrm{Ti}(>0.10-0.30)$, higher $\mathrm{Zr} / \mathrm{Y}$ $(>2),(\mathrm{Ce} / \mathrm{Yb})_{\mathrm{N}}<1$, and $(\mathrm{La} / \mathrm{Sm})_{N}>1$.
The occurrence of extremely depleted lavas in the Garrett transform could suggest the presence of an anomalous mantle underneath the fracture zone. However similar trends of basaltic depletion are observed in the Siqueiros transform [Natland, 1989; Perfit et al., 1994], and the systematic association of anomalous primitive mantle with transforms is difficult to explain. Also, in the Siquieros transform, Natland [1989] reported that enriched and depleted MORBs are found in proximity. A second hypothesis is that the source for the Garrett volcanics constitutes the residue left from a previous SEPR mantle source. For the same reason as above, this hypothesis implies a complex mechanism for preferentially segregating this type of depleted mantle in transform faults. This would suggest that only depleted melts are able to form in the Garrett transform. Another, more reasonable explanation would be to assume that the mantle source in the transform is similar to that of the EPR ridge axis but that the thermal regime differs. Thermal differences could affect the style of melting and the shallow magmatic processes thus giving rise to the preferential extrusion of depleted lavas.

The concept of multistage melting of a composite mantle [Hékinian et al., 1989] permits the progressive melting of mantle during upwelling, giving rise to a variety of melts going from enriched to depleted MORBs on ridge axis. In this case, the compositional differences observed between the NEPR (more E-MORB) and the SEPR could be explained by slight differences in their respective sources (less enriched for the SEPR) or more probably by more efficient mixing in shallow level magmatic reservoir (more buffering composition in SEPR than in the NEPR) (Figure 4). From recent work on the clinopyroxene composition in lherzolites [Johnson et al., 1990], it is assumed that partial melting of the mantle is probably perfectly fractional (no porosity) or near-fractional (i.e., with some porosity). The difference between these two modes of partial melting is significant for the composition of the solid residues and less so for that of the melt. Therefore a simple nonmodal fractional melting model (Raleigh fractionation) with melt accumulation was adopted as a first approximation to estimate the composition of produced liquids and to compare these with the observed basalt compositions (Figures 8a and 8b). The accumulated melt composition as a function of the amount of melting is derived from the following equation [Shaw, 1970]: $C M=C S / F[1-(1-P F / D)\}(1 / P)$

where $C M$ is the integrated partial melt composition, $C S$ is the concentration of the trace element in the initial solid (Table 4), $F$ is the mass fraction of the melt, $D$ is the bulk rock partition coefficient, and $P$ is the partition coefficient of the melting mineral phases. $P$ is constrained using the mass fractions of the melting minerals as derived from stoichiometric coefficients estimated from laboratory melting experiments on dry peridotites [Kinzler and Grove, 1992a, b]. The coefficients used correspond to the average value calculated for a spinel lherzolites at $12 \mathrm{kbar}$ [Kinzler and Grove, 1992a] and at 10 kbar [Falloon and Green, 1983] (Table 4). These coefficients are kept constant over the entire range of melting.

The Ronda peridotite (R255), having suitable trace elements as well as modal analyses, was choosen from among the available lherzolites, as the main mantle source for the Garrett volcanics. However this particular spinel lherzolite is already depleted in clinopyroxene (73\% olivine, $20 \%$ orthopyroxene, and 7\% clinopyroxene). Prinzhofer et al., [1989] proposed a lherzolitic source including clinopyroxen- 
A

Garrett (basalts)

A

L1, L2, L3=liquid produced during step melting
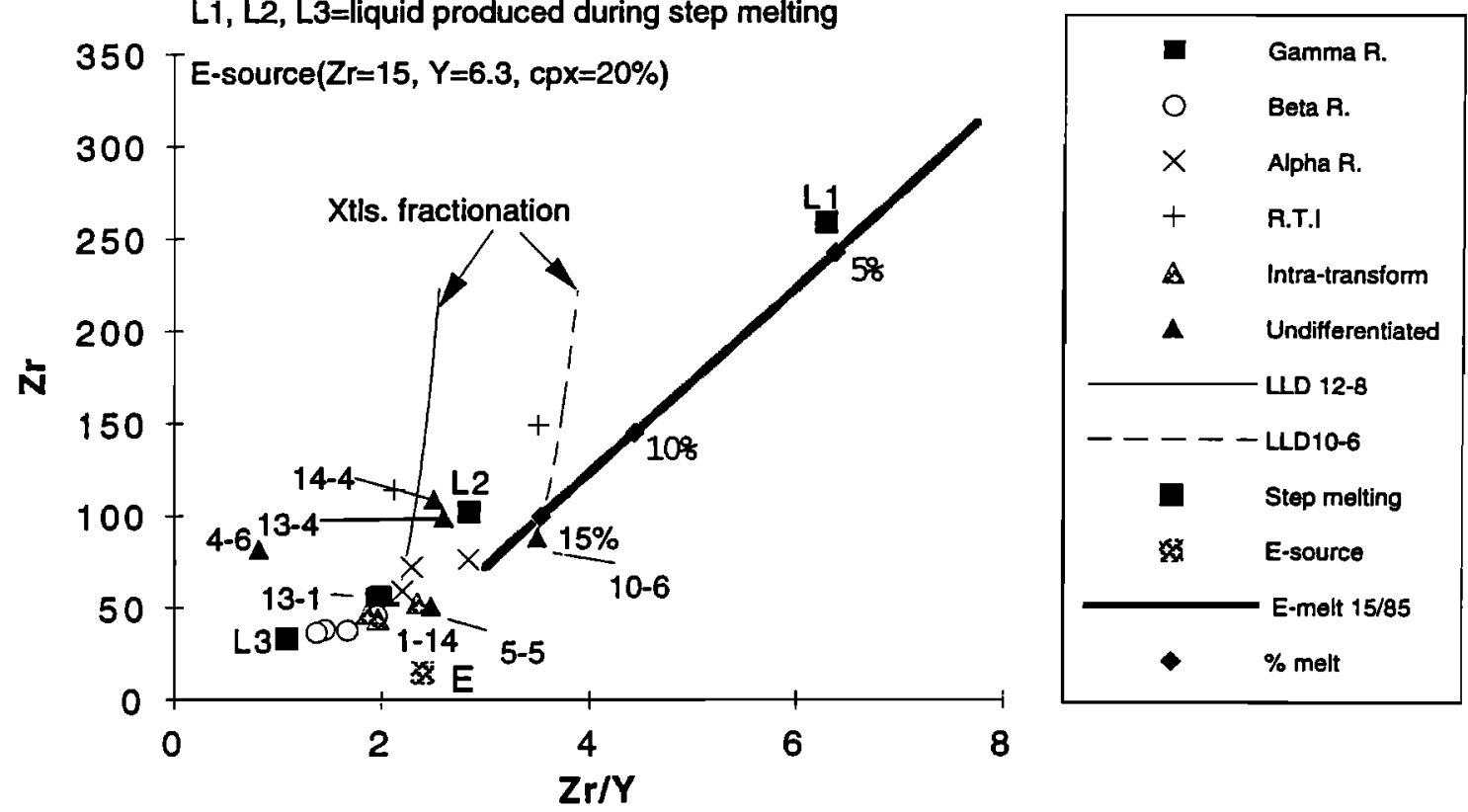

B

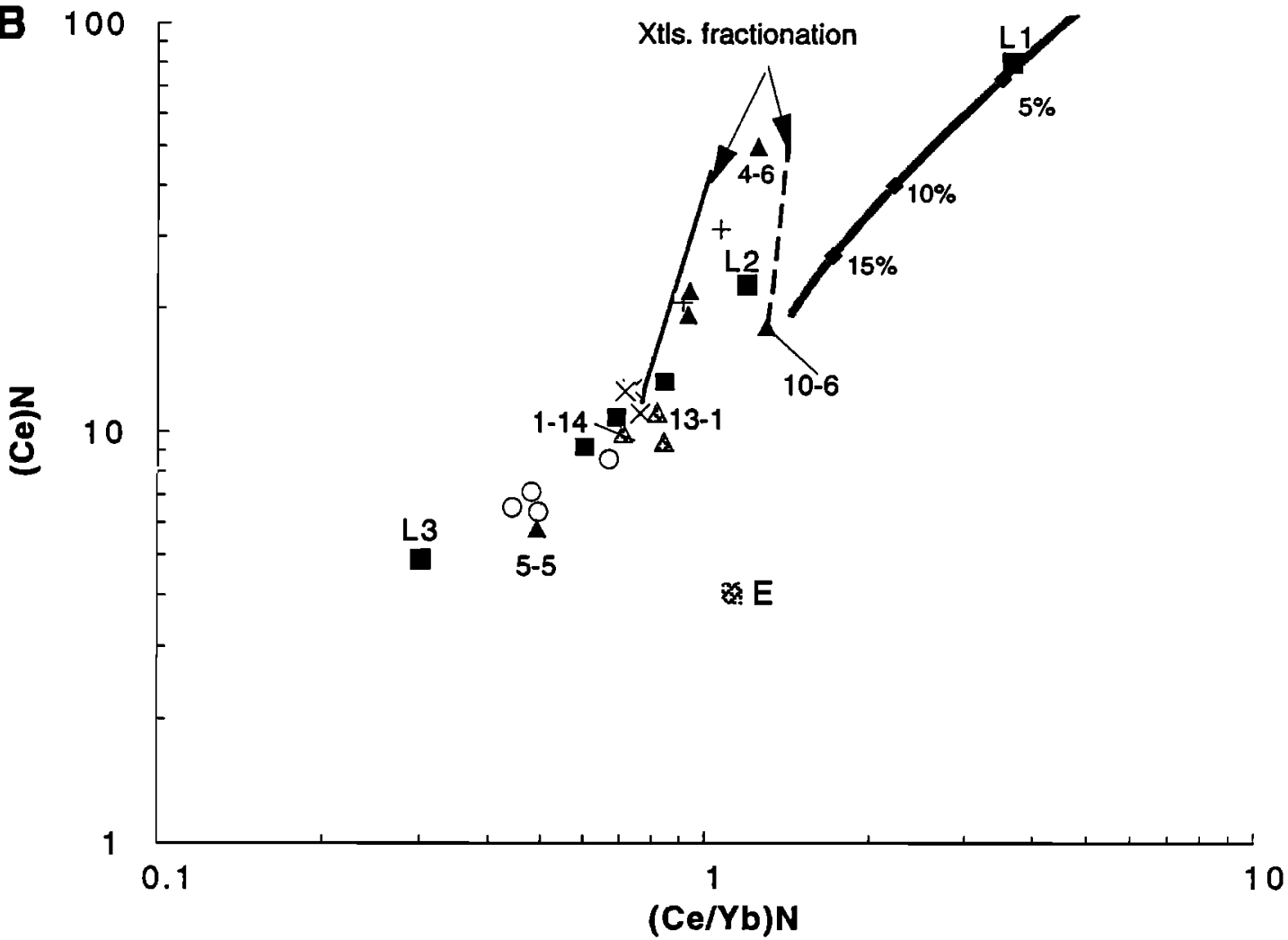

Figure 8. (a) $\mathrm{Zr}-\mathrm{Zr} / \mathrm{Y}$ and (b) $(\mathrm{Ce})_{N}-(\mathrm{Ce} / \mathrm{Yb})_{N}$ (normalized to chondrite $\left.\mathrm{C} 1\right)$ [Sun and McDonough, 1989] variation diagrams of basalts from the Garrett transform and the ridge-transform intersection (RTI) zone. An enriched mantle source $(\mathrm{Zr}=15 \mathrm{ppm}, \mathrm{Y}=6.3 \mathrm{ppm}$, clinopyroxene=20\%) was chosen for nonmodal fractional melting models (Table 4). In this case, each source represents a residue left during successive depletion during stepwise partial melting of a composite mantle [Hékinian et al., 1989; Bideau and Hékinian, this issue] (also see text). The liquids (L1, L2, and L3) were produced by three-step melting stages during near-fractional melting (incremental melting of $1 \%$ ) process, assuming that the permeability threshold is $5 \%$ after each magma extraction [Bideau and Hékinian, this issue]. A total melting of less than $15 \%$ is sufficient to produce the most depleted liquid (L1). The liquid lines of descent (LLD) are calculated from the least evolved melts (12-8 and 10-6). The symbols are the same as in Figures $3 a$ and $3 b$. 
Table 4a. Mantle Sources and Partition Coefficients Used for Partial Melting Models of Mid-Ocean Ridge Basalts

\begin{tabular}{|c|c|c|c|c|c|}
\hline Minerals & $\begin{array}{c}\text { Lherzolite } \\
\text { R255 } \\
\text { D Source }\end{array}$ & $\begin{array}{c}\text { Clinopyroxenite } \\
\text { SC73-2P } \\
\text { E-source }\end{array}$ & $\begin{array}{l}\text { Mixed } \\
15 / 85\end{array}$ & $\begin{array}{l}\text { Stochiometric } \\
\text { coefficient }\end{array}$ & $\begin{array}{c}\text { Proportion } \\
\text { of Melting } \\
\text { Phases } \\
(20 \%)\end{array}$ \\
\hline $\begin{array}{l}\text { Olivine } \\
\text { Orthopyroxene }\end{array}$ & $\begin{array}{l}0.710 \\
0.200\end{array}$ & $\begin{array}{l}0.02 \\
0.000\end{array}$ & $\begin{array}{l}0.600 \\
0.170\end{array}$ & $\begin{array}{c}-0.3 \\
0.4\end{array}$ & $\begin{array}{l}-0.046 \\
0.08\end{array}$ \\
\hline Clinopyroxene & 0.070 & 0.090 & 0.200 & 0.82 & 0.164 \\
\hline Spinel & 0.020 & 0.080 & 0.030 & 0.08 & 0.016 \\
\hline $\mathbf{Z r}$ & 6 & 66 & 15 & & \\
\hline $\mathbf{Y}$ & 3 & 25 & 6.3 & & \\
\hline $\mathbf{K}$ & 83 & 664 & 170 & & \\
\hline $\mathrm{Ti}$ & 700 & 11630 & 2339 & & \\
\hline $\mathbf{L a}$ & 0.30 & 3.46 & 0.77 & & \\
\hline $\mathrm{Sm}$ & 0.26 & 4.13 & 0.84 & & \\
\hline $\mathrm{Ce}$ & 0.78 & 12.20 & & & \\
\hline Yb & 0.38 & 1.96 & 0.62 & & \\
\hline$(\mathrm{Ce})_{N}$ & 1.27 & 19.93 & 4.07 & & \\
\hline$(\mathrm{Ce} / \mathrm{Yb})_{N}$ & 0.57 & 1.73 & 1.12 & & \\
\hline $\mathrm{Zr} / \mathrm{Y}$ & 2.00 & 2.64 & 2.38 & & \\
\hline $\mathrm{K} / \mathrm{Ti}$ & 0.11 & 0.06 & 0.07 & & \\
\hline
\end{tabular}

Sources are spinel-lherzolite R255, Frey et al. [1978] and spinel-clinopyroxenite SC73-2P, Irving [1980]. E Source is $15 \% \mathrm{SC73/2P}+85 \%$ RC255. D source is spinel lherzolite from Frey et al. [1978], depleted in clinopyroxene. Stochiometric coefficients are from Kinzler and Grove [1992a].

ite as a fertile component for the NEPR near $13^{\circ} \mathrm{N}$ lavas. For the purpose of the present work, a composite source was simulated in order to obtain about $20 \%$ clinopyroxene. This composite source model is composed of $85 \%$ spinel lherzolite from the Ronda high-temperature peridotite (R255) [Frey et al., 1985] and 15\% spinel-clinopyroxenite from Hawaian xenoliths (Sc73-2P) [Irving, 1980]. The resulting source candidate contains $64 \%$ olivine, $17 \%$ orthopyroxene, and $20 \%$ clinopyroxene (Table 4). We have combined the clinopyroxene from the two sources into a single homogeneous phase for the purpose of calculation. About $24 \%$ partial melting of the composite source is necessary to completely remove the clinopyroxene, and at $15 \%$ melting, the mass fractions of the mineral phases are close to that of the spinel lherzolite R255.

The melting curves calculated for $(\mathrm{Ce})_{N}$ versus $(\mathrm{Ce} / \mathrm{Yb})_{N}$ and for $\mathrm{Zr}$ versus $\mathrm{Zr} / \mathrm{Y}$ enclose the enriched MORBs and part of the depleted MORBs of the SEPR samples (Figures $8 \mathrm{a}$ and $8 \mathrm{~b}$ ). The most enriched (E-MORBs) samples are found to occur on the NEPR $\left(11^{\circ} 26^{\prime}\right.$ and $\left.13^{\circ} \mathrm{N}\right)$ (Figures $9 \mathrm{a}$ and $9 \mathrm{~b}$ ). Otherwise, most GITV are strongly depleted $\left[\mathrm{Zr} / \mathrm{Y}<2.5 ;(\mathrm{Ce} / \mathrm{Yb})_{N}<1\right]$ with respect to the depleted basalts from the NEPR and SEPR segments. This model helps us to understand the production of enriched SEPR and NEPR lavas as well as some less enriched volcanics (samples 10-6, 13-4, and 14-4) from transform domains. However, the model fails to explain the origin of the other more depleted MORBs from the GITV. Some of the

Table 4b . Partition Coefficients Used for Partial Melting Models of Mid Ocean Ridge Basalts

\begin{tabular}{|c|c|c|c|c|c|c|c|c|c|c|}
\hline Mineral & $\mathbf{Z r}$ & $\mathbf{Y}$ & $\mathbf{K}$ & $\mathbf{T i}$ & $\mathbf{N i}$ & $\mathbf{L a}$ & Sm & $\mathrm{Nb}$ & $\mathrm{Ce}$ & $\mathrm{Yb}$ \\
\hline Plagioclase & 0.01 & 0.03 & 0.01 & 0.01 & 0.01 & 0.027 & 0.200 & 0.01 & 0.001 & 0.05 \\
\hline Olivine & 0.010 & 0.010 & 0 & 0 & 10 & 0.003 & 0.003 & 0.01 & 0.001 & 0.059 \\
\hline Orthopyroxene & 0.020 & 0.200 & 0.01 & 0.1 & 4 & 0.009 & 0.026 & 0.15 & 0.005 & 0.075 \\
\hline Clinopyroxene & 0.107 & 0.510 & 0.025 & 0.44 & 3 & 0.05 & 0.50 & 0.10 & 0.1 & 0.5 \\
\hline
\end{tabular}

Sources are $\mathrm{Zr}$, Johnson et al. [1990], Pearce and Norry [1979], averages from Hékinian et al. [1989]; Y, Pearce and Norry [1979], averages from Hékinian et al., [1989]; K, Pearce and Norry [1979]; Ni, Frey et al. [1974, 1978]; Ti, Johnson et al. [1990] andPearce and Norry [1979]; La, Chen and Frey [1985], Green and Pearson [1985], and Ottonello [1980]; Sm, Johnson et al. [1990] and Prinzhofer and Allègre [1985]; Ce, Prinzhofer and Allègre [1985], Johnson et al. [1990], Tarney et al. [1980], averages from Hékinian et al. [1989]; Yb, Prinzhofer and Alléère [1985], Johnson et al. [1990], averages from Hékinian et al. [1989]. 
A Garrett-EPR

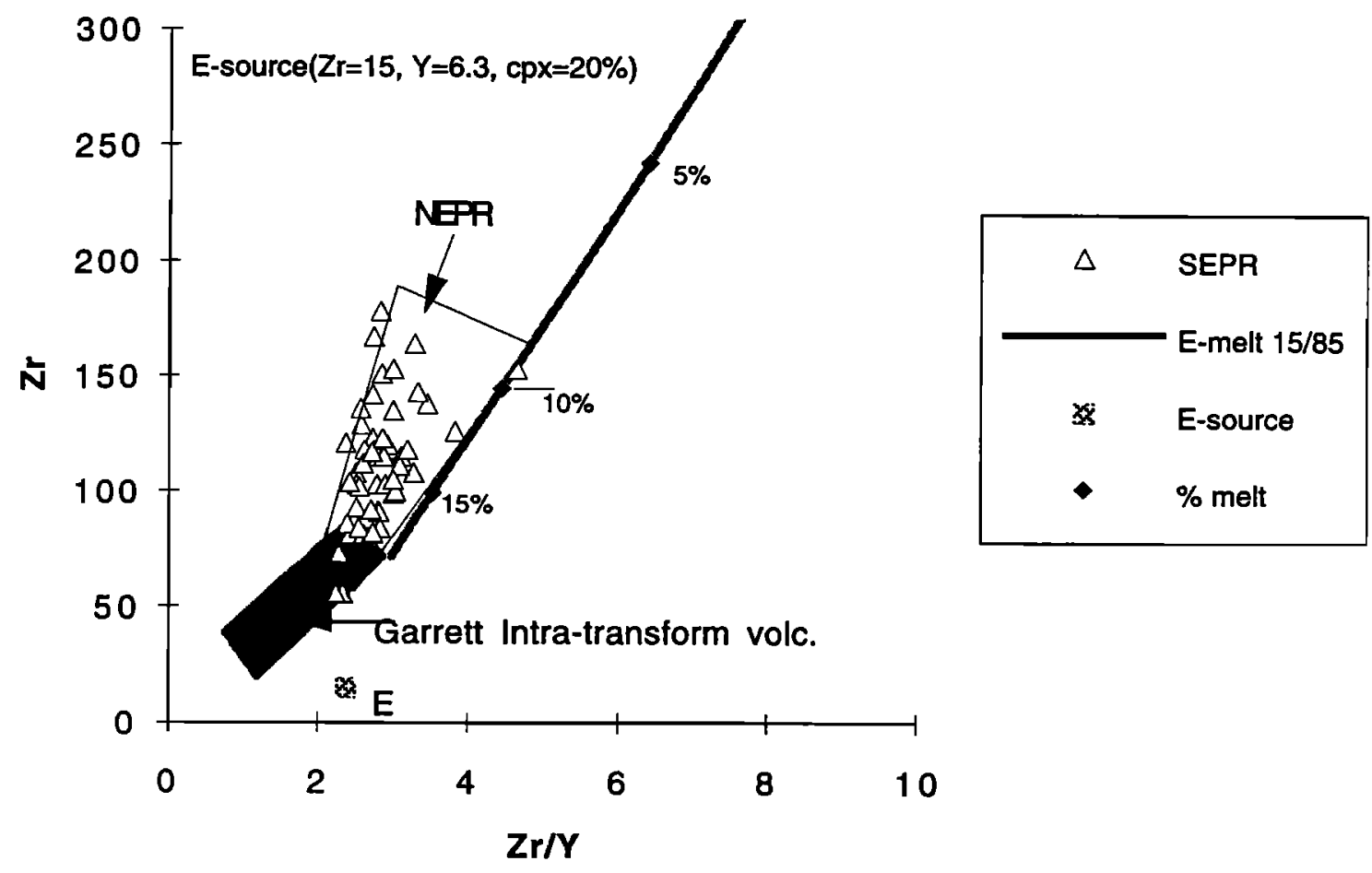

B

Garrett-EPR

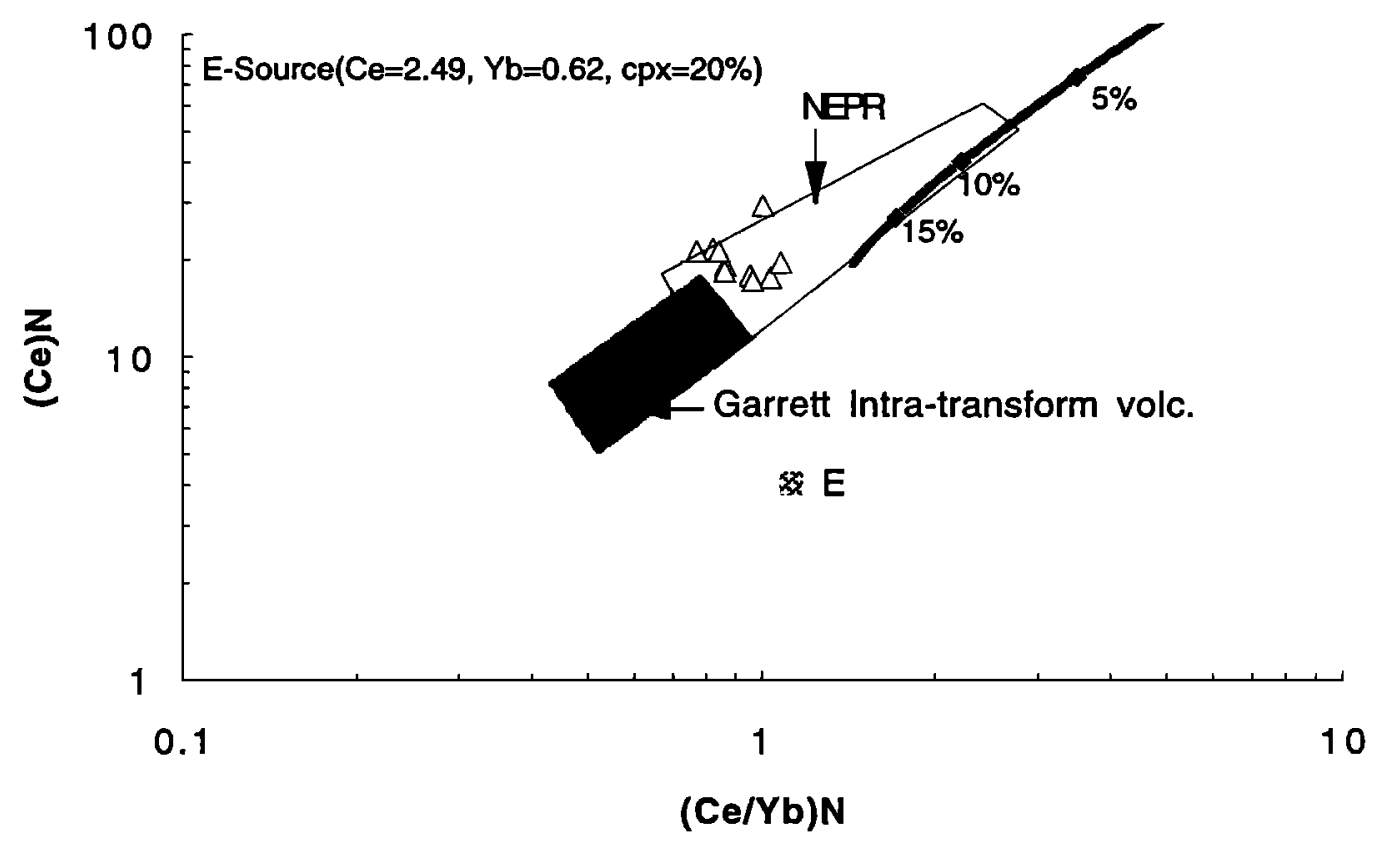

Figure 9. (a) $\mathrm{Zr}-\mathrm{Zr} / \mathrm{Y}$ and (b) $(\mathrm{Ce})_{N-}(\mathrm{Ce} / \mathrm{Yb})_{N}$ variation diagrams of basalts from the north East Pacific Rise (NEPR) and the south East-Pacific Rise (SEPR) axial segments compared to the volcanics from Garrett transform. The field for the Garrett intratransform (GITV) volcanics includes only the young flows from oblique ridges and intratransform troughs and wall. The liquid lines of descent (LLD) were calculated for the EMORB sample DR3-16 from the NEPR at $12^{\circ} 43^{\prime} \mathrm{N}$ [Hékinian et al., 1989]. The NEPR $\left(9^{\circ} \mathrm{N}-21^{\circ} \mathrm{N}\right)$ data are from Février [1981], Allan et al. [1989], Fornari et al. [1988], Thompson et al. [1989] and Hékinian et al. [1989]. The SEPR $\left(17^{\circ} 26^{\prime}\right.$ and $21^{\circ} 26^{\prime}$ S) data are from Table 3 and from Bach et al. [1994] and Mahoney et al. [1994] for $\mathrm{Zr}$ and $\mathrm{Y}$ values. The symbols are the same as in Figure 8a. 

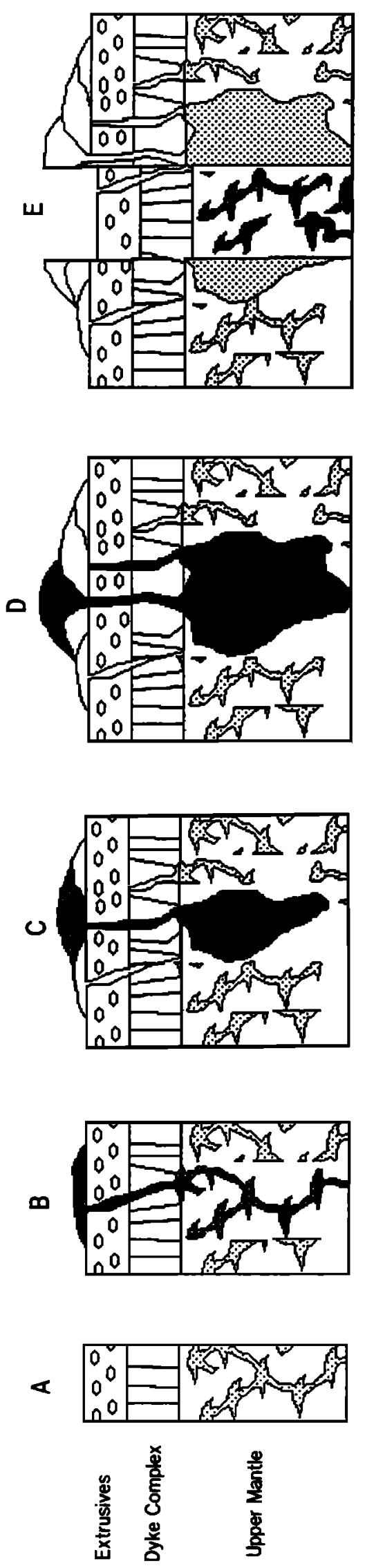

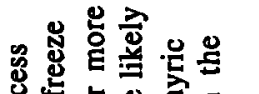

samples from the GITV are off the main trends of the melting curves and consist of aphyric basalts (7-1, 7-7, and 7-8) from the Beta ridge, which are extremely depleted (Figures 8a and $8 b)$. Since the residual solid resulting from $20 \%$ partial melting of the composite source is harzburgitic in composition, there are no stoichiometric coefficients available to extend the melting curve to the depleted MORBs of the Garrett samples. We therefore have used a multistage melting and extraction process derived from the present model and developed elsewhere by Bideau and Hekinian [this issue]. This model succeeds in producing liquids having an appropriate degree of depletion for fitting the composition of the GITV for less than $15 \%$ total melting of the mixed source used. For the Garrett intratransform volcanics a three-step melting stage was adopted (Figure 8a). In this hypothesis, the partial melting of a composite mantle starts with the production of enriched melt and terminates with that of a depleted melt at the end of a magmatic cycle (Figures 10a and 10b). In order to provide contrasting melt composition, the process of melt segregation at depth should be discontinuous and proceed by successive steps of melt accumulation separated by rapid extraction. However, this model does not explain the exclusive occurrence of depleted lavas reaching the seafloor, and it is believed that other shallow level processes partly related to the thermicity of the upper mantle-crust region are involved in limiting the extrusion of E-MORBs in the Garrett transform.

\section{Thermal Boundary Effects}

The cold edge effect of propagators and transform fault zones could enhance a contrasting composition of erupted lava in going from rather primitive to highly differentiated basalts, such as the ferrobasalts found in the RTI region [Christie and Sinton, 1981; Hey et al., 1989]. It is also well known that at the tip of propagators [Christie and Sinton, 1981; Hekinian et al., 1994], where the cooling rates are the highest, the lavas tend to be as unevolved and as porphyritic as those found in Garrett. The Garrett intratransform volcanics have undergone a limited extent of crystal fractionation (about $20 \%$ on average). They consist of less evolved and generally more porphyritic basalts than other fast and ultrafast EPR volcanics. In addition, all the extruded lava on the transform floor consists essentially of extremely depleted MORBs.

If the Bideau and Hékinian [this issue] model is applicable to Garrett samples, the early enriched melt end-member must freeze in the upper mantle or lower crust rather than be extruded on the seafloor (Figure 10a). The progressive reheating of the lithosphere by these early intrusions and a limited extent of mixing should favor the exzrusion of parental melts having compositions near those of the depleted end-members (Figure 10b). The hypothesis that part of the early melts produced in the Garrett transform did not reach the ocean floor but instead froze in the lithosphere before extrusion is supported by the observations of abundant wehrlitic and gabbroic impregnations in the Garrett peridotites [Hekinian et al., 1992; Cannat et al., 1990]. These melt impregnations could partly result from the freezing of trapped liquids and partly from reaction with early melt fractionations migrating through the uppermost mantle on their way toward the surface.

The ascent of magma through narrow fissures or dykes in the fast cooling environment of the Garrett transform should 


\section{GARRETT INTRA-TRANSFORM MAGMATISM}

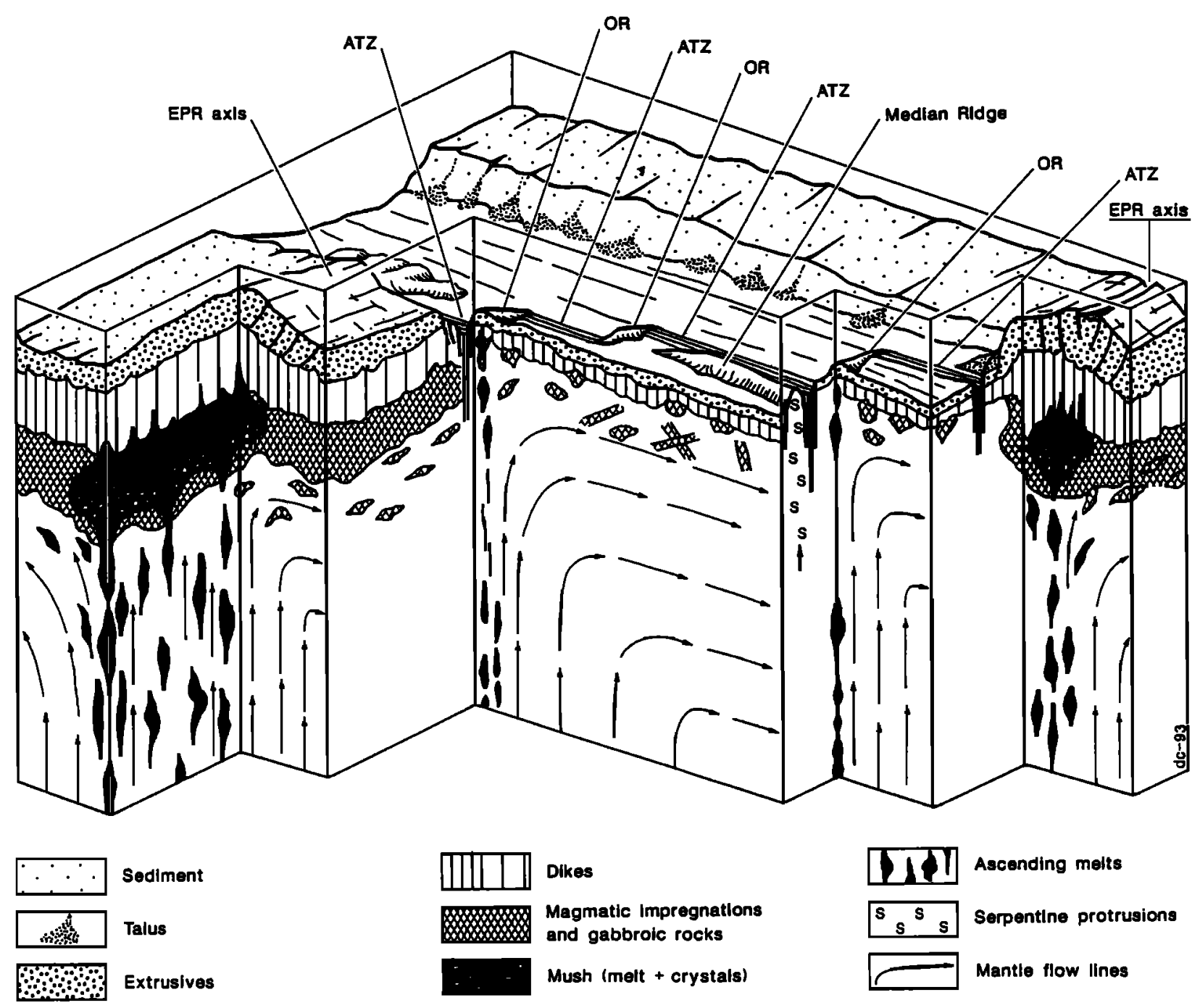

ATZ - Active Tectonic Zone

OR = Oblique Fldges

Figure 11. Sketched block diagram representing contrasted types of magmatic activities taking place between the lithosphere forming the Garrett transform and that of the nearby ultrafast East Pacific Rise (EPR) segments. While limited blobs of magmatic injections are associated with the intratransform volcanism, more steady state shallow reservoirs characterize the EPR axial segments where mixing and crystal fractionation occur. Discontinuous magma supply within a cool oceanic lithosphere, such as in the Garrett transform, will enhance the formation of short-lived and small-sized pockets and/or conduits of magma loaded with early formed crystals (viscous magma) and give rise to the eruption of phyric primitive lava undergoing limited extent of mixing (Figures 10c and 10d).

increase its viscosity as well as crystal nucleation rates and therefore prevent a large extent of crystal settling, leading to preferential extrusions of highly porphyritic lavas (Figure 10c). The first lava extruded on the transform floor should consist of viscous rather unevolved flows containing large amounts of crystals (highly porphyritic lavas). However, the formation of small-sized and short-lived magma chambers or conduits could follow, allowing for small extent of fractional crystallization to take place (Figure 10d). During these later conditions, a limited mixing occurred as attested to by the oc- casional presence of abnormal crystal zoning [Hébert et al., 1994] which is indicative of low diffusion rates and nonequilibrium mixing processes. At the end of a magmatic cycle (or at ridge-transform intersection zones) the lithosphere might remain hot enough to permit the extrusion of highly fractionated aphyric lavas (ferrobasalts) in the absence of magmatic replenishment (Figure 10d). In contrast with the NEPR volcanics, the more homogeneous and generally less enriched nature of the basalts erupted on the SEPR axis implies a larger extent of mixing between the two end-member melts (enriched 
and depleted) in a long-lived and more steady state magma chamber (Figure 11). For a similar mantle source composition, the petrological differences observed between the SEPR segments and the Garrett intratransform volcanics are likely to be controlled by the spatial and temporal variations of the thermal budget within the lithosphere.

\section{Conclusion}

More than $25 \%$ of the Garrett transform domain has been the site of volcanic activity taking place at more than $3500 \mathrm{~m}$ depth. The lava erupted in the Garrett active transform domain mainly consists of depleted basalts (D-MORBs) with low $\mathrm{K} / \mathrm{Ti}$ ( $<0.1), \mathrm{Zr}(<120 \mathrm{ppm}), \mathrm{Ba}(<110 \mathrm{ppm}), \mathrm{Zr} / \mathrm{Y}$ (1.4-2.3), and $(\mathrm{Ce} / \mathrm{Yb})_{N}(0.3-0.7)$. Rare occurrences of transitional (TMORB) $(\mathrm{K} / \mathrm{Ti}=0.14-0.25, \mathrm{Ba}=8-18 \mathrm{ppm}, \mathrm{Zr} / \mathrm{Y}=2.5-3.5$, and $(\mathrm{Ce} / \mathrm{Yb})_{N=0.9-1.3)}$ basalts found on older volcanic terrains of the Garrett transform are believed to represent south East Pacific Rise (SEPR) volcanics. The most depleted rocks consist of olivine and plagioclase phyric basalts except for the samples collected from the EPR-transform intersection zone (RTI). These later samples are ferrobasalts containing early clinopyroxene crystallizing with a more sodic plagioclase and Mg-poor olivine (Fo55) and are characterized by high $\mathrm{TiO}_{2}(1.8-2.5 \%)$ and $\mathrm{FeO}(>11 \%)$ contents. The ferrobasalts and the T-MORBs are believed to represent SEPR crust rather than outcrops of the Garrett oblique ridges or recent intratransform trough and wall volcanics (GITV) as indicated from field observations.

Crystal-liquid fractionation explains some of the variation observed among samples belonging to certain magmatic lineages. While an average of about $20 \%$ of crystallization (mainly plagioclase) is necessary to obtain many of the evolved samples, the ferrobasalts are believed to be produced by more than $30 \%$ crystallization. With the exception of the ferrobasalts from the RTI, the Garrett intratransform volcanics (GITV) show less variation for $\mathrm{K} / \mathrm{Ti}(<0.14)$ and the $\mathrm{Mg} \#(>0.58)$ than those from other SEPR segments. The oblique ridge basalts have lower $\mathrm{Zr}(<70 \mathrm{ppm})$ and generally higher Ni ( $>100 \mathrm{ppm}$ ) contents than those from the southern EPR segments $\left(13^{\circ}-21^{\circ} \mathrm{S}\right)$. Even if some crystal fractionation has taken place, the intratransform melts have had very little residence time in the lithosphere and/or were solidified in restricted individual conduits rather than in large magmatic reservoirs.

Volcanics from different oceanic provinces, such as the northern East Pacific Rise (NEPR) segments at $21^{\circ} \mathrm{N}, 13^{\circ} \mathrm{N}$, and $11^{\circ} 26^{\prime} \mathrm{N}$, contain a wider range of incompatible elements $\left(\mathrm{Zr}=60-200 \mathrm{ppm}\right.$, and $\left.\mathrm{Zr} / \mathrm{Y}=2-5,(\mathrm{Ce} / \mathrm{Yb})_{N}=0.6-13\right)$ and $\mathrm{K} / \mathrm{Ti}$ ratios (0.05-0.5) than do the samples from Garrett. The strong depletion in incompatible elements for the Garrett intratransform volcanics with respect to that of the accreting plate boundary of other SEPR $\left(13^{\circ} 30^{\prime}-21^{\circ} \mathrm{S}\right)$ and NEPR segments suggests that the Garrett samples were emplaced in a cool lithospheric environment with only a limited amount of mixing.

The volcanics from the Garrett transform are believed to have been derived from the partial melting of a composite mantle during a multistage melting and melt extraction process. This same model could also applied to the origin of the EPR volcanics. This model developed elsewhere [Bideau and Hekinian, this issue] and adopted here could explain the extremely depleted nature of the Garrett intratransform vol- canics, without invoking a different source from that of the SEPR. However, in order to account for the restricted range of compositional variabilities, it is assumed that most of the enriched end-member lavas did not reach the surface but rather were trapped as impregnation gabbros and wehrlites at the mantle-crust transition zone.

Acknowledgments. We are thankful to the captain, the officers, and the crew of the R/V Nadir as well as to the Nautile team for their help and enthusiasm expressed during the cruise. The microprobe analyses referred to in the text were performed with the technical help of Marcel Bohn on a Camebax SX50 at IFREMER. The bulk chemical analyses were done at the Centre de Recherche Petrographique et Géochimique de Nancy (France) by using spectrometric methods coupled to a plasma (ICP) source, under the supervision of J. Morel and $K$. Govindaraju. The Garrett cruise was sponsored and organized by IFREMER. We are thankful to R. Batiza, W. B. Bryan, G. Bluth, R.S. Detrick, P. Michael, and anonymous reviewer (JGR) for their constructive comments, which helped to improve the manuscript.

\section{References}

Allan J. F., R. Batiza, M. R. Perfit, D. J. Fornari, and R. O. Sack, Petrology of lavas from the Lamont seamount chain and adjacent East Pacific Rise, 10N, J. Petrol. , 30, 1245-1298, 1989.

Bach, W., E. Hegner, J. Erzinger, and M. Satir, Chemical and isotopic variations along the superfast Spreading East Pacific Rise from 6 to 305, Contrib. Mineral. Petrol., 116, 365-380, 1994.

Backer, H., J. Lange, and V. Marchig, Hydrothermal activity and sulphide formation in axial valleys of the East Pacific Rise crest between $18^{\circ} \mathrm{S}$ and $22^{\circ} \mathrm{S}$, Earth, Planet. Sci. Lett., 72, 9-22, 1985.

Batiza, R., and Y. Niu, Petrology and magma chamber processes at the East Pacific Rise near 9³0'N, J. Geophys. Res., 97, 6779-6797, 1992.

Bender, J. F., C. H. Langmuir, and G. N. Hanson, Basalt glasses from the Tamayo region, East Pacific Rise, J. Petrol., 25. 213-254, 1984.

Bideau, D., and R. Hékinian, A dynamic model for generating smallscale heterogeneities in ocean floor basalts, J. Geophys. Res., this issue.

Bonatti, E. Subcontinental mantle exposed in the Atlantic Ocean on St. Peter-Paul Islets, Nature, 345, 800-802, 1990.

Cannat, M., D. Bideau, and R. Hébert, Plastic deformation and magmatic impregnation in serpentinized ultramafic rocks from the Garrett transform fault (East Pacific Rise), Earth, Planet. Sci. Lett., 101, 216-232, 1990.

Casey, J.F., D. J. Fornari, M. R. Perfit, W. I. Ridley, and C. Xia, Alvin diving along strike slip faults linking intratransform spreading centers in the Siqueiros Transform Domain: Documentation of plutonic exposures and evidence for "leakage" along a PTDZ, EOS Trans. $A G U, 72$ (44), Fall Meeting supply, 525-526, 1991.

Chen, C.Y., and F. A. Frey, Trace elements and isotopic geochemistry of lavas from haleakala volcano, East Maui, Hawaii: Implications for the origin of Hawaiian basalts, J. Geophys. Res., 90, 8743-8768, 1985.

Christie, D. M., and J. M. Sinton, Evolution of abyssal lavas along propagating segments of the Galapagos Spreading Center, Earth Planet. Sci. Lett., 56, 321-335, 1981.

Clague, D. A., and T. E. Bunch, Formation of ferrobasalt at East pacific mid-ocean spreading centers, J. Geophys. Res., 81, 4247-4256, 1976.

Eissen, J.-P., D. Bideau, and T. Juteau, Présence de basaltes porphyritiques dans les zones de fractures de la dorsale Est-Pacifique, $C . R$. Acad. Sci., Ser. 2, 293, 61-66, 1981.

Falloon, T. J., and D. H. Green, Anhydrous partial melting of MORB pyrolite and other peridotite compositions at $10 \mathrm{kbar}$. Implication for the origin of primitive MORB glasses, Mineral. Petrol., 37, 181-219, 1983.

Février, M., Hydrothermalisme et minéralisation sur la dorsale Est 
Pacifique à $21^{\circ} \mathrm{N}$, thèse 3ème cycle, 270 pp., Univ. Bretagne Occidentale et Cent. Natl. pour l'Exploitat. des Océans, Brest, 1981.

Fornari, D. J., R. Batiza, R. Haymon, A. Barone, W. B. F. Ryan T. Smith, T. Simkin, and M. Luckman, Geochemical and structural studies of the Lamont seamounts: Seamounts as indicators of mantle processes, Earth Planet. Sci. Lett., 89, 63-83, 1988.

Fomari, D. G., D. G. Gallo, M. H. Edwards, J. A. Madsen, M. R. Perfit, and N. Shor, Structure and topography of the Siqueiros transform fault system: Evidence of intratransform spreading centers, Mar. Geophys. Res., 11, 263-299, 1989.

Fox, P. J., and D. G. Gallo, Transform of the Eastern Central Pacific, in The Geology of North America, vol N, The Eastern Pacific Ocean and Hawaii, edited by E. L. Winterer, D. M. Hussong, and R. W. Decker, pp. 111-124, Geological Society of America, Boulder, Colo., 1989.

Frey, F. A., W. B. Bryan, and G. Thompson, Atlantic floor: Geochemistry and petrology of basalts from legs 2 and 3 of the Deep-Sea Drilling Project, J. Geophys. Res., 79, 5507-5527, 1974.

Frey, F. A., D. H. Green, and S. D. Roy, Integrated models of basalt petrogenesis: A study of quartz tholeiites to olivine melilites from southeast Australia utilizing geochemical and experimental petrologic data, J. Petrol., 19, 463-513, 1978.

Frey, F. A., C. J. Suen, and H. W. Stockman, The Ronda high temperature peridotite: Geochemistry and petrogenesis,. Geochim. Cosmochim. Acta, 49, 2469-2491, 1985.

Govindaraju, $K$., Compilation of working values and sample description for 272 geostandards, Geostand. News, 13, spec. issue, 1-113, 1989.

Green, T. H., and N. J. Pearson, Rare element partitioning between clinopyroxene and silicate liquid at moderate to high pressure, Contrib. Mineral Petrol., 91, 24-36, 1985.

Hébert, R., D. Bideau, and R. Hékinian, Ultramafic and mafic rocks from the Garrett transform fault near $13^{\circ} 30^{\prime} S$ on the East Pacific Rise: Igneous petrology, Earth Planet. Sci. Lett., 65, 107-125, 1983.

Hébert, R., R. Hékinian, and D. Bideau, Petrology of a new class of magmatic activity: Intra-transform volcanism in the Garrett transform, Earth Planet. Sci. Lett., in press, 1995.

Hékinian, R., G. Thompson, and D. Bideau, Axial and off-axial heterogeneity of basaltic rocks from the East Pacific Rise at $12^{\circ} 35^{\prime} \mathrm{N}$ $12^{\circ} 51^{\prime} \mathrm{N}$ and $11^{\circ} 26^{\prime} \mathrm{N}-11^{\circ} 30^{\prime} \mathrm{N}$, J. Geophys. Res., $94,17437-17463$, 1989.

Hékinian, R., D. Bideau, M. Cannat, J. Francheteau, and R. Hébert, Volcanic activity and crust-mantle exposure in the ultrafast Garrett transform fault near $13^{\circ} 28^{\prime} \mathrm{S}$ in the Pacific, Earth Planet. Sci. Lett., 108, 259-275, 1992.

Hey, R. N., J. M. Sinton, and F. K. Duennebier, Propagating rifts and spreading centers, in The Geology of North America, vol. N, The Eastern Pacific Ocean and Hawaii, edited by E. L. Winterer, D. M. Hussong, and R. W. Decker, pp. 161-176, Geological Society of America, Boulder, Colo., 1989.

Irving, A. J., Petrology and geochemistry of composite ultramafic xenoliths in alkalic basalts and implications for magmatic processes within the mantle, Am. J. Sci., 280-A, 389-426, 1980.

Johnson, K. T. M., H. J. B. Dick, and N. Shimizu, Melting in oceanic upper mantle: An ion microprobe study of diosides in abyssal peridotites, J.. Geophys. Res., 95 , 2661-2678, 1990.

Kinzler R., and T. L. Grove, Primary magmas of mid-ocean ridge basalts, 1,. Experiments and methods, J. Geophys. Res., 97, 68856906, 1992a.

Kinzler, R. J., and T. L. Grove, Primary magmas of mid-ocean ridge basalts, 2, Applications, J. Geophys. Res., 97, 6907-6926, 1992b.

Langmuir, C., J. F. Bender, and R. Batiza, Petrological and tectonic segmentation of the East Pacific Rise, $5^{\circ} 30^{\prime} \mathrm{N}-14^{\circ} 30^{\prime} \mathrm{N}$, Nature, 322 , 422-427, 1986.

Lonsdale, P., Near bottom reconnaissance of a fast-slipping transform fault zone at the Pacific-Nazca plate boundary, J. Geol., 6, 451-472, 1978.
Lonsdale, P., Segmentation of the Pacific-Nazca spreading center, $1^{\circ} \mathrm{N}-20^{\circ} \mathrm{S}, \mathrm{J}$. Geophys. Res. 94, 12,197-12,225, 1989.

Mahoney, J. J., J. M. Sinton, M. D. Kurz, J. D. Macdougall, K. J. Spencer, and G. W. Lugmair, Isotope and trace elements characteristics of a super-fast spreading ridge: East Pacific rise, $13-23^{\circ} \mathrm{S}$, Earth Planet. Sci. Lett., 121, 173-193, 1994.

Melson, W. G., E. Jarosewich, and R. Cifelli, Alkali olivine basalt dredged near St Paul's rocks, Mid-Atlantic Ridge, Nature, 215, 381382, 1967.

Melson, W. G., S. R. Hart, and G. Thompson, St Paul's Rocks Equatorial Atlantic petrogenesis, radiometric ages, and implications on seafloor spreading, Mem. Geol. Soc. Am., 132, 242-272, 1972.

Natland, J. H, Partial melting of a lithologically heterogeneous mantle: Inferences from crystallization histories of magnesian abyssal tholeiites from the Siqueiros fracture zone, in magmatism in the Ocean Basins, edited by A. D. Saunders and M. J. Norry, Geol, Soc. Spec. Publ. London, 42, 41-70, 1989.

Ottonello, G., Rare earth abundances and distribution in some spinel peridotite xenoliths from Assab (Ethiopia), Geochim. Cosmochim. Acta, 44, 1885-1901, 1980.

Pearce, J. A., and M. J. Norry. Petrogenetic implications of Ti, Zr, Y, and $\mathrm{Nb}$ variations in volcanic rocks, Contrib. Mineral. Petrol., 69, 33-47, 1979.

Perfit, M. R., et al., Recent volcanism in the Siqueiros Transform Fault: Eruption of picritic and High-MgO basalts and implication for MORB magma genesis, Earth Planet. Sci. Lett. , in press, 1995.

Prinzhofer, A., and C. J. Allègre, Residual peridotites and the mechanisms of partial melting, Earth Planet. Sci. Lett., 74, 251-265, 1985.

Prinzhofer, A., E. Lewin, and C. J. Allègre. Stochastic melting of the marble cake mantle: Evidence from local study of the East Pacific Rise at $12^{\circ} 50$ N, Earth Planet. Sci. Lett., 92, 189-206, 1989.

Renard, V., R. Hékinian, J. Francheteau, R. D. Ballard, and H. Backer, Submersible observations at the axis of the ultra-fast-spreading East Pacific Rise (17 $30^{\prime}$ to $21^{\circ} 30^{\prime}$ S), Earth Planet. Sci. Lett., 75, 339-353, 1985.

Searle, R., Multiple, closely spaced transform faults in fast-slipping fracture zones, Geology, 1I, 607-610, 1983.

Shaw, D. M., Trace element fractionation during anatexis, Geochim. Cosmochim. Acta, 34, 237-243, 1970.

Sinton, J. M., S. M. Smaglik, J. J. Mahoney, and K. C. Macdonald, Magmatic processes at superfast spreading oceanic ridges: Glass variations along the East Pacific Rise, $13^{\circ}-23^{\circ} \mathrm{S}, J$. Geophys. Res., 96, 6133-6155, 1991.

Sun, S.-S., and W. F. McDonough, Chemical and isotopic systematics of oceanic basalts: Implications for mantle composition and processes, in Magmatism in the Ocean Basins , edited by A.D. Saunders and M.J. Norry, Geol. Soc. Spec. Publ. London, 42, 313-345, 1989.

Thompson, G., W. B. Bryan, R. D. Ballard, K. Hamuru, and W. G. Melson, Axial processes along a segment of the East Pacific Rise, $10-12^{\circ} \mathrm{N}$, Nature, 318, 429-433, 1985.

Thompson, G., W. B. Bryan, and S. E. Humphris, Axial volcanism on the East Pacific Rise, $10-12^{\circ} \mathrm{N}$, in Magmatism in Ocean Basins, edited by A.D. Saunders and M.J. Norry, Geol. Soc. Spec. Publ. London, 42, 181-200, 1989.

Weaver, J. S., and C. H. Langmuir, Calculation of phase equilibrium in mineral melt systems, Comput. Geosci., 16(1), 1-19, 1990.

D. Bideau and R. Hékinian, IFREMER, Centre de Brest, B.P. 70, 29280 Plouzané cedex, France. (e-mail: dbideau@ifremer.fr.; hekinian@ifremer.fr)

R. Hébert, Département de Géologie, Université Laval, Pavillon Adrien Pouliot, Quebec, Canada. G1K 7P4.

Y. Niu, Department of Earth Science, University of Queensland, Brisbane, Queensland 4072, Australia.

(Received March 14, 1994; revised July 8, 1994; accepted August 11, 1994.) 